\title{
Functional imaging of mucociliary phenomena
}

\section{High-speed digital reflection contrast microscopy}

\author{
M. Ryser • A. Burn · Th. Wessel · M. Frenz • \\ J. Rička
}

Received: 6 December 2006/Revised: 27 February 2007/Accepted: 1 March 2007/Published online: 17 April 2007

(C) EBSA 2007

\begin{abstract}
We present a technique for the investigation of mucociliary phenomena on trachea explants under conditions resembling those in the respiratory tract. Using an enhanced reflection contrast, we detect simultaneously the wave-like modulation of the mucus surface by the underlying ciliary activity and the transport of particles embedded in the mucus layer. Digital recordings taken at a speed of 500 frames per second are analyzed by a set of refined data processing algorithms. The simultaneously extracted data include not only ciliary beat frequency and its surface distribution, but also space-time structure of the mucociliary wave field, wave velocity and mucus transport velocity. Furthermore, we propose the analysis of the space and time evolution of the phase of the mucociliary oscillations to be the most direct way to visualize the coordination of the cilia. In particular, this analysis indicates that the synchronization is restricted to patches with varying directions of wave propagation, but the transport direction is strongly correlated with the mean direction of waves. The capabilities of the technique and of the data-processing algorithms are documented by characteristic data obtained from mammalian and avine tracheae.
\end{abstract}

Keywords Cilia $\cdot$ Ciliary beat frequency $\cdot$ Metachronal wave $\cdot$ Ciliary coordination $\cdot$ High-speed video microscopy $\cdot$ Metachronal wavelength . Mucociliary transport

Electronic supplementary material The online version of this article (doi:10.1007/s00249-007-0153-3) contains supplementary material, which is available to authorized users.

M. Ryser · A. Burn · Th.Wessel · M. Frenz · J. Rička ( $ه)$

Institute of Applied Physics, University of Bern,

Sidlerstrasse 5, 3012 Bern, Switzerland

e-mail: rick@ fluor.iap.unibe.ch

\section{Introduction}

Cilia are hair-like extensions of the cell membrane that cover the surface of many protozoan cells or inner surfaces in various parts of the vertebrate body. The most striking feature of these extracellular organelles are their swift shimmering motions, which have fascinated observers since Leeuwenhoeks first observations of Vorticella and since the discovery of the ubiquity of these Flimmerhärchen by Purkinje and Valentin (1834). Driven by molecular motors fueled by ATP (Satir and Sleigh 1990), the cilia oscillate with a frequency of $15-30 \mathrm{~Hz}$ (Joki and Saano 1994). The purpose of this activity is to achieve transport: locomotion of protozoa or transport of body fluids in higher organisms. The most prominent location of cilia in the body is the bronchial tract, where they participate in the "'mucociliary escalator", a sophisticated mechanism for cleaning inhaled particles from the lung (Sleigh et al. 1988; Wanner et al. 1996). The particles are trapped in a viscoelastic mucus layer covering the epithelium and the mucus carpet is propelled by the beating cilia. It is obvious that in order to achieve an efficiently directed transport the ciliary motions must be, at least locally, coordinated. Indeed, ciliated surfaces exhibit coordinated beat patterns resulting from a well-defined shift in the beat phase between individual cilia, the so-called "metachronal waves". Metachronal waves are easily observable on ciliated protozoa, e.g., on the pretty opalina, and have been described by early researchers (Gray 1930). In the case of respiratory cilia, the coordination and its relation with transport are considerably less well understood (if at all), despite much research in this direction (Sleigh et al. 1988; Wanner et al. 1996). The main reason for our limited knowledge is the lack of observations under native conditions in the respiratory tract, which is only partly compensated by the remarkable 
ability of respiratory cilia to maintain their activity in explants for many hours post-mortem. Even in excised trachea samples observation of undisturbed ciliary activity is difficult. The contrast is poor, because of the dense packing of the respiratory cilia (6-8 cilia per square micrometer) and because the observation is only possible through the corrugated mucus layer above an irregularly formed epithelium topology.

The most conspicuous feature of the ciliary activity, namely the regular beating, can be measured relatively easily (this only applies ex vivo). Since the first attempts to measure the ciliary beat frequency (CBF) by cinematography (Lucas 1932), numerous techniques have been developed and continue to be developed, boosted by the recent surge in the performance of opto-electronic devices and in available computing power. The contemporary techniques for $\mathrm{CBF}$ measurements can be roughly divided into two classes. The first class includes variants of the socalled dynamic light scattering technique (DLS) (Lee and Verdugo 1976; Bonnaire et al. 1980; Svartengren et al. 1989; Chandra et al. 1994; Wessel et al. 1999; Bogdanovic et al. 2005): one illuminates the sample by coherent laser light and observes fluctuations of the random interference pattern (speckles) resulting from the more or less random superposition of light waves scattered or reflected in the ciliated epithelium. The advantage of the DLS techniques is the simplicity of the optical setup and of the data processing. For example, in our variant (Wessel et al. 1999) the setup consists of two single-mode optical fibers: one delivers the laser beam and the other collects the scattered light. A photon-counter receives the scattered light and processing is done on-line using a digital correlator. DLS can be also done in imaging mode by observing the fluctuations of a speckle pattern with a fast digital imager (Bogdanovic et al. 2005). The second class of CBF techniques employs standard white light imaging, usually with differential interference contrast (Hennessy et al. 1986; Teichtahl et al. 1986; Ben-Shimol et al. 1991; Romet et al. 1991; Curtis et al. 1991; Dresdner and Wong 1985; Rautiainen et al. 1992; Sanderson 2000; Chilvers and O'Callaghan 2000; Sisson et al. 2003; Dimova et al. 2005; Schipor et al. 2006). Since affordable digital imagers with frame rates larger than $200 \mathrm{~Hz}$ became available, they have become a must for this type of CBF measurements. The advantage of the imaging approach is the spatial resolution of the CBF determination. Moreover, the information content of the digital video images is high, allowing a deeper space-time analysis of the ciliary activity than mere CBF measurements.

The goal of characterizing the space-time features of respiratory ciliary activity, i.e., the metachronal wave field, is considerably more difficult to achieve than the simple CBF determination. Much of today's knowledge about the respiratory metachronicity is highlighted in the work by Sanderson and Sleigh (1981) on rabbit tracheal epithelium: "The metachronal waves are usually said to be only of a short wavelength, extending over few cells, and to move generally in an antiplectic fashion. The distance traveled is also small, the wave tending to fade." Sanderson and Sleigh confirmed this picture in an ingenious, albeit somewhat indirect way, by fixation of the metachronal wave and subsequent electron-microscopic observation. (Their attempt to observe the metachronicity directly by high-speed cinematography seems to have largely failed because of the lack of contrast).

An extensive series of experiments aimed at the quantitative characterization of the synchronization of the beating cilia in a living cell culture was undertaken by Priel and co-workers (1987; Ovadyahu and Priel 1989; Gheber and Priel 1989, 1994). These researchers worked mostly with transparent-cultured frog palate epithelium, using cleverly arranged fiber optic probes to detect the light transmitted through the cell layer. From the cross-correlation of the signals from probes at varying spacing they were able to measure the wavelength as well as the velocity of the metachronal waves. Essentially, they confirmed the classical picture, i.e., antiplectic metachronism with correlation distances restricted to few tens of micrometers. The small values of the metachronal wavelength they measured $(5-9 \mu \mathrm{m})$ may be a property of the particular investigated cell culture. A similar cross-correlation technique has been reported by Wong et al. (1993), who used a light scattering system with a spatially alternating focal spot instead of several fibers. From the cross-correlation of the two alternating signals they obtained what they call the "metachronal wave period".

Recently, studies aiming at the space-time features of the mucociliary activity became rare, as the interest shifted to the underlying chemical signaling mechanisms (Korngreen and Priel 1994; Salathe and Bookman 1995; Sanderson et al. 1990). A notable exception is the recent work by Yi et al. (2002, 2003) and Lee et al. (2005), who employed standard digital video microscopy (at $30 \mathrm{~Hz}$ frame rate) and determined the spatially resolved CBF and the socalled "metachronal wave disorder" by analyzing the gray value fluctuations of the digitized video frames.

We are convinced that the possibilities of digital imaging for the elucidation of mucociliary phenomena are by no means exhausted. In the present work we introduce a technique designed for the investigation on trachea explants under conditions that resemble as closely as possible the native conditions in the respiratory tract. Using an enhanced reflection contrast technique, which is free of the orientation artifacts associated with traditional Nomarski contrast, we detect the modulation of the mucus surface by the underlying ciliary activity and the transport 
of tracer particles embedded in the mucus layer simultaneously. Digital recordings taken at a speed of 500 frames per second are analyzed by a set of refined data processing algorithms. The simultaneously extracted data include CBF and its surface distribution, space-time structure of the modulation wave field, wave velocity and mucus transport velocity. The background motivation for the technical developments was a comprehensive interspecies study on mammalian (bovine, porcine, ovine, lapine) and avine tracheae (turkey, ostrich). The results of these studies are presented in an accompanying publication (Burn et al., submitted). Here we focus on the technical aspects of data acquisition and evaluation, using selected representative data from this systematic study as illustrative examples.

\section{Experimental procedures}

Sample and climate chamber

Our samples are freshly excised tracheae from mammals (bovine, porcine, ovine and lapine) and birds (turkey and ostrich) obtained from the local slaughterhouse. The tracheae were transported inside a sealed and thermally isolated bag to our lab and stored in a refrigerator at $4^{\circ} \mathrm{C}$. The measurements always took place the same day. During the process of sample preparation we meticulously avoided touching the mucus layer covering the ciliary epithelium in order not to disturb the mucociliary apparatus. For the same reason the samples were cut out as large as practically convenient, at least several square centimeters. The samples consisted of the entire trachea wall, including the cartilage tissue. We sprayed a small amount of tracers onto the mucus layer to facilitate the observation of mucus transport. Puff ball spores with a diameter of $3.5 \mu \mathrm{m}$ served well for this purpose. Finally, the trachea pieces were fixed into specially designed sample-holders to flatten the observed surfaces and were subsequently placed into the precisely controllable climate chamber, as sketched in Fig. 1. For all measurements, the trachea pieces were mounted onto the microscope stage with the pharynx-end pointing up (away from the observer). This convention was maintained throughout the whole analysis process and is also used in all graphics shown. The climate chamber consists of a piece of flexible polyurethane membrane sealed around the sample holder and the microscope objective (see Fig. 1). The sample holder is placed on a tiltable stage which allows adjustment of the tilt of the irregular sample surface. The temperature of the stage is stabilized by means of a heating foil, temperature controller module and a Pt-100 sensor embedded in the stage. A second temperature sensor is fixed on the sample surface to measure the temperature of the epithelium. The massive aluminum stage and firm fixation guaranteed a homogeneous temperature distribution. Good heat conductance between the temperature-controlled stage and the sample was assured by filling possible air-gaps between tissue and sample holder with serum free medium (OptiMEM I, GIBCO/Invitrogen, Carlsbad, CA, USA). All presented data were taken when the temperature remained stable at $30^{\circ} \mathrm{C}$ with a tolerance of $\pm 0.1^{\circ} \mathrm{C}$. We preferred to work at comparatively low sample temperature of $30^{\circ} \mathrm{C}$ in order to minimize drying and to avoid condensation. With an inflow of water-saturated air the relative humidity was kept higher than $95 \%$ during the measurements.

\section{Imaging setup}

The experimental setup is built around an upright microscope (Nikon Eclipse E600FN). Since we wish to observe collective phenomena of a tissue area that encompasses many ciliated cells, a low magnification objective is sufficient. With the 10x objective (Nikon Plan Fluor, NA 0.3 , WD $16 \mathrm{~mm}$ ) we image an area of $0.5 \times 0.5 \mathrm{~mm}^{2}$ onto the chip of one of the two following digital cameras: (1) Dalsa CA-D1, frame grabber Epix PIXCI ${ }^{\circledR}$ D R3.0 (max. frame rate 495 fps, dynamic range $\approx 1: 1000,128 \times 128$ pixels, field of view $456 \times 456 \mu \mathrm{m}$ ), (2) Dalsa CA-D6, frame grabber Epix PIXCI ${ }^{\circledR} \mathrm{D} 32$ R0.0, $(1,000 \mathrm{fps}$, dynamic range $\approx 1: 256,256 \times 256$ pixels, field of view $563 \times 563 \mu \mathrm{m}$ ). The advantage of the DALSA CA-D1 is the high sensitivity and large dynamic range, useful for the observation of low contrast samples. On the other hand, the Dalsa CA-D6 system provides a larger field of view and better resolution for detailed analysis of the trachea surface. (At the time of manuscript preparation this imaging hardware was already obsolete. The cameras and frame-grabbers can be replaced by affordable and simple-to-use FireWire devices such as DragonFly Express by Point Grey Research.) The choice of the image acquisition speed is dictated by the swiftness of the ciliary motions: According to Nyquist's sampling theorem the sampling frequency must be at least twice the maximal frequency component one wishes to observe. A standard video camera delivers 25-50 frames per second, which is barely sufficient for a reliable determination of the ciliary oscillation frequency in the range $10-20 \mathrm{~Hz}$. Because we want to observe the details of the space-time properties of the metachronal wave field, we prefer working at much higher frame rates of $200-500 \mathrm{~Hz}$. The decrease of the information content with decreasing frame rate and increasing integration time can be seen even in the still pictures in Fig. 2. To capture at least 15 cycles of the ciliary beat we recorded sequences with a minimum of 512 frames, i.e., at least $1 \mathrm{~s}$. 


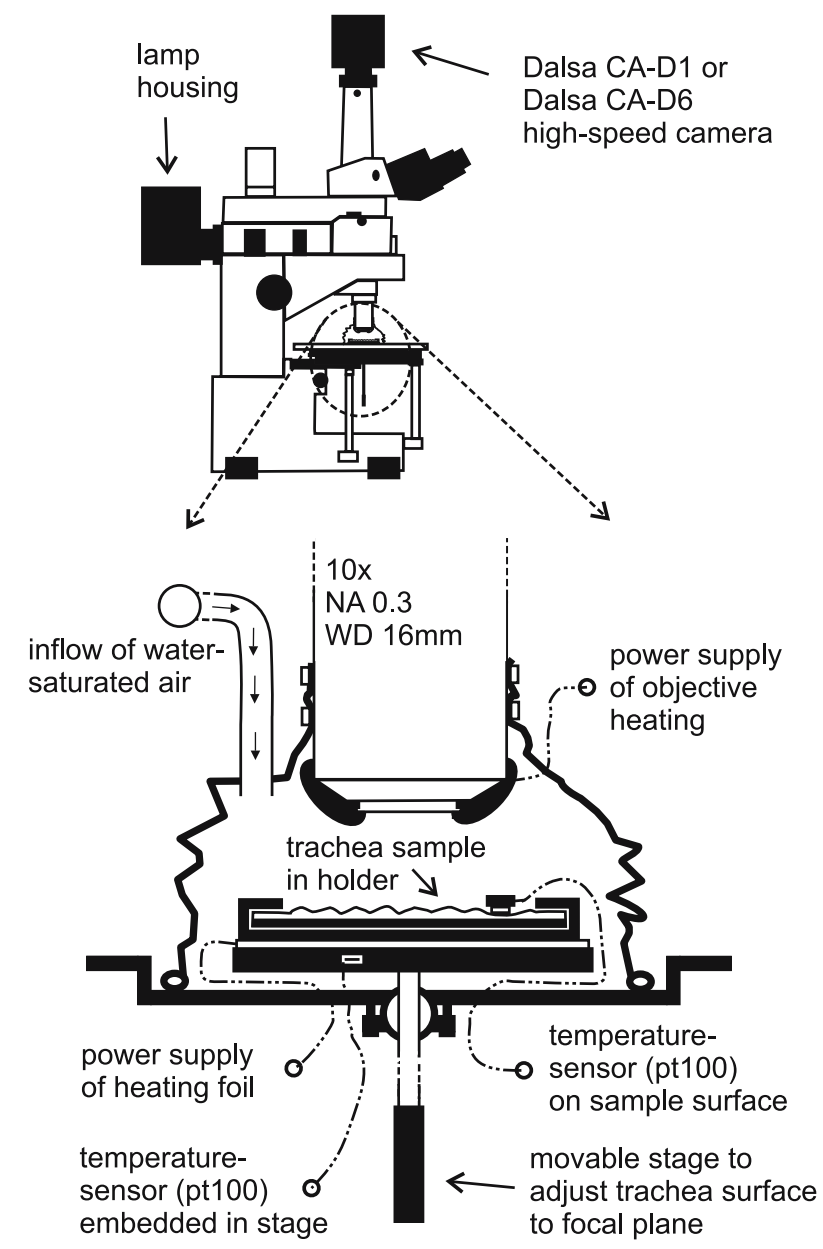

Fig. 1 Details of the trachea sample placed in the climate chamber

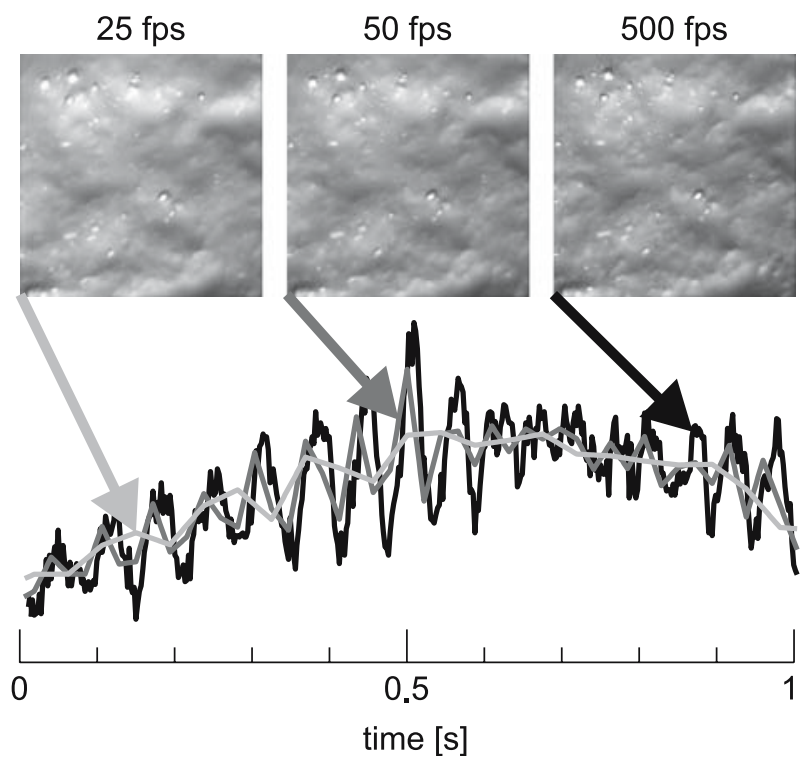

Fig. 2 Top Effect of frame rate on the image quality. Bottom Signal evolution in one pixel as it would appear at three different frame rates. The data at lower frame rates were generated by averaging the original high resolution movie, recorded at $500 \mathrm{fps}$
Illumination and contrast

Crucial for the observation of metachronal phenomena is a technique providing adequate contrast. The excised trachea samples are very limited in their transparency and the observation by transmission light microscopy from the apical side is therefore not practicable. We illuminate the sample from above, through the microscope objective, using a standard Hg-fluorescence lamp equipped with Köhler optics. (To minimize radiant heating of the sample, the near-IR content of the lamp is blocked by an appropriate filter.) With epi-illumination, the dominant contribution to the received light originates from the reflection at the air-water (air-mucus) interface. Scattering from the densely packed cilia layer and diffuse scattering from the underlying tissue are negligible compared with the specular reflection at the interface. Consequently, the only source of useful contrast is the modulation of the air-mucus surface by the beating cilia. Traditionally, the contrast technique of choice used in such a situation is Nomarski Differential Interference Contrast (DIC) microscopy (Wessel et al. 1999; Rautiainen et al. 1992; Schipor et al. 2006). The DIC technique, however, is not suitable for quantitative analysis of the metachronal wave-field because of an inherent imaging artifact: the image structure depends on the orientation of the sample with respect to a Wollaston prism. Fortunately, a simple reflection contrast technique is sufficient for imaging of mucus modulation, as can be seen in the example in Fig. 2. Qualitatively, reflection contrast can be understood using simple geometrical optics, as indicated in Fig. 3.

A detailed quantitative analysis of reflection contrast will be given elsewhere. Here we only note that the luminance distribution in a reflection contrast image of a weakly modulated surface can be well approximated by the following semi-empirical formula:

$I(x, y) \propto \exp \left(-4 \frac{\beta(x, y)^{2}}{\alpha^{2}}\right)$,

where $\beta(x, y)^{2}=z_{x}(x, y)^{2}+z_{y}(x, y)^{2} . \beta$ is the inclination angle as defined in Fig. 3. The first order partial derivatives $z_{x}(x, y)$ and $z_{y}(x, y)$ are the gradient components representing the local slope of the surface. The parameter $\alpha$ is proportional to the numerical aperture of the observation. Thus, by inserting an appropriate diaphragm in the back focal plane of the objective, one can adjust the contrast to the desired level: decreasing the aperture will improve the visibility of the surface modulation due to the beating cilia, but it may exaggerate the static features of the trachea topography. 

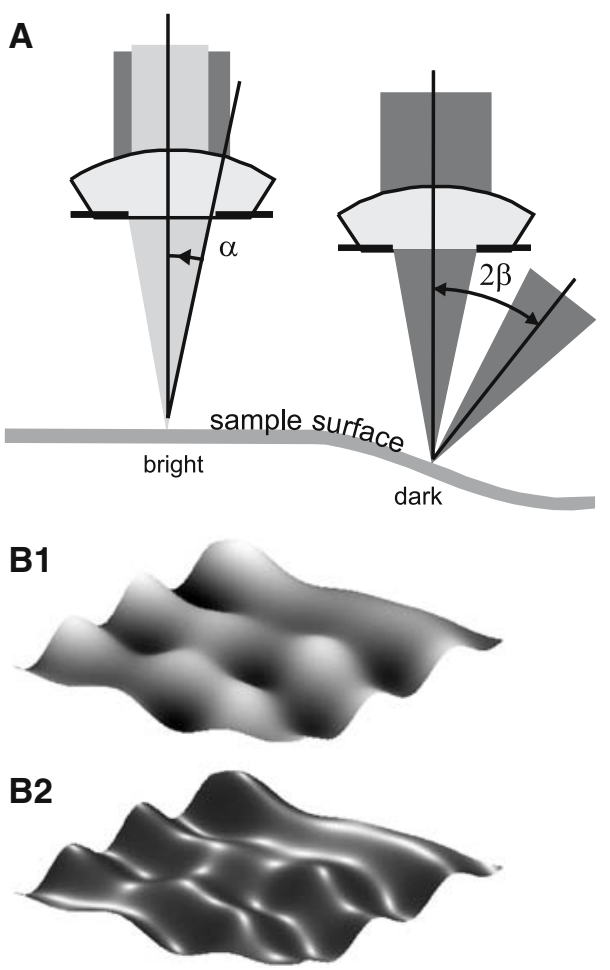

Fig. 3 a Principle of reflection contrast. The rays of the illuminating light impinging on a flat, horizontal surface are reflected back into the acceptance angle $\alpha$ of the objective, but those which hit a steep enough slope are deflected so that $\beta>\alpha$ and are thus lost for observation. Therefore flat, horizontal surfaces appear bright and slopes more or less dark; the image is completely dark when the tilt angle $\beta$ exceeds the aperture angle $\alpha$. b1 The gray-values on the modulated surface are coded according to the surface height. b2 Gray values coded as they appear in reflection contrast

The exact value of $\alpha$ depends on the geometry of the illumination (e.g., on the size of the Köhler light source) and is best determined by calibration using a reference surface, such as a well polished and clean steel ball. Once $\alpha$ is known, one can in principle attempt a reconstruction of the surface profile $z(x, y)$ by inverting Eq. 1 . An example is illustrated in Fig. 4.

The image appearance suggests a simple plane wave structure with surface gradient pointing along the marked line. Thus we can try a one-dimensional reconstruction from the corresponding luminosity profile. Lacking the sign of the inclination angle $\beta$, the reconstruction cannot be unique. We are forced to make reasonable assumptions: (1) the sign of the gradient only changes at local maxima or minima. (2) In order to preserve the spatial frequency only single or pair changes of the gradient signs at consecutive local extrema are allowed. Three possible surface profiles satisfying these assumptions are shown in the lower graph in Fig. 4. Each of the profiles shows a characteristic behavior, but we cannot decide with certainty which actually represents the topology of
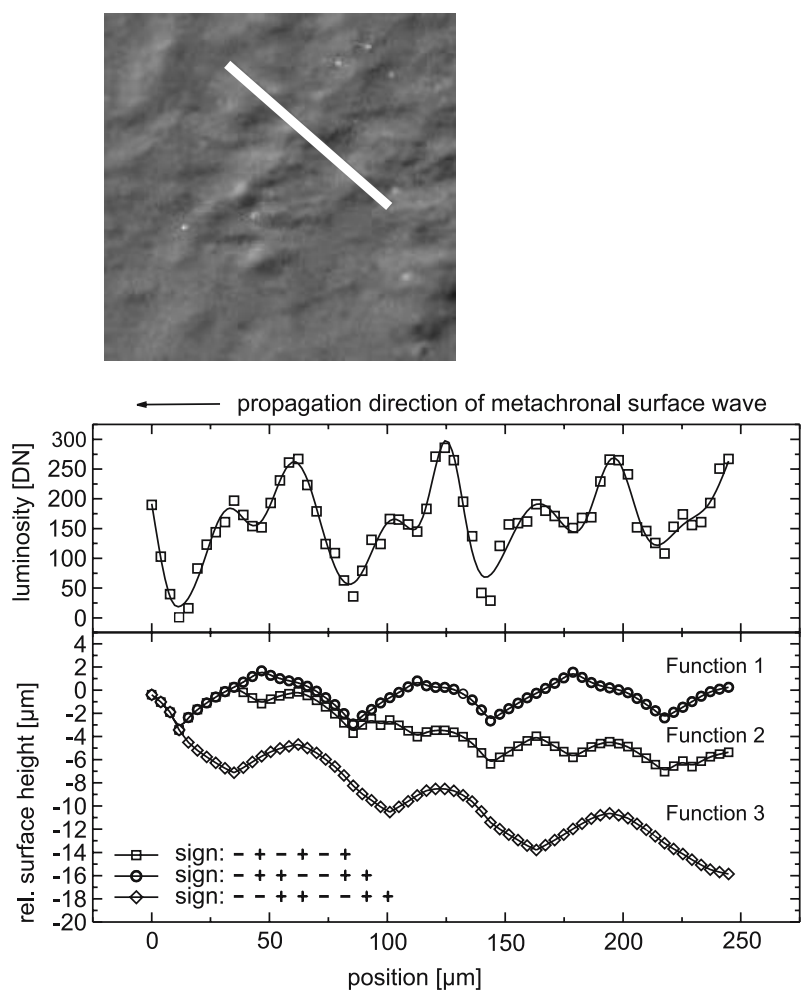

Fig. 4 Luminosity along a line in an image from a bovine trachea sample (top graph) and three reconstructed surface profiles (lower graph)

the specimen. Because it is the smoothest, function 3 seems to be the most likely one. Common to all profiles is a height variation of $4-5 \mu \mathrm{m}$ corresponding to about $50 \%$ of the total length of a cilium. It is obvious that such reconstruction only works in favorable circumstances of especially smooth trachea topography and simple structure of the surface modulation. Thus, the present example only serves to demonstrate the sensitivity of the reflection contrast technique.

\section{Preprocessing}

A raw reflectometry image, such as the example in Fig. 5a, contains three types of information:

1. Tracer particles transported with the mucus.

2. Surface modulation due to the beating cilia, which we call "metachronal wave field".

3. Static features of the trachea topography.

We are only interested in features (1) and (2), which we wish to extract from the raw image. First, we extract the tracers. Since the tracers are small point-like objects, the extraction can be done using a local filtering technique. The classical Lee-filter (Lee 1986) serves well our purpose. 


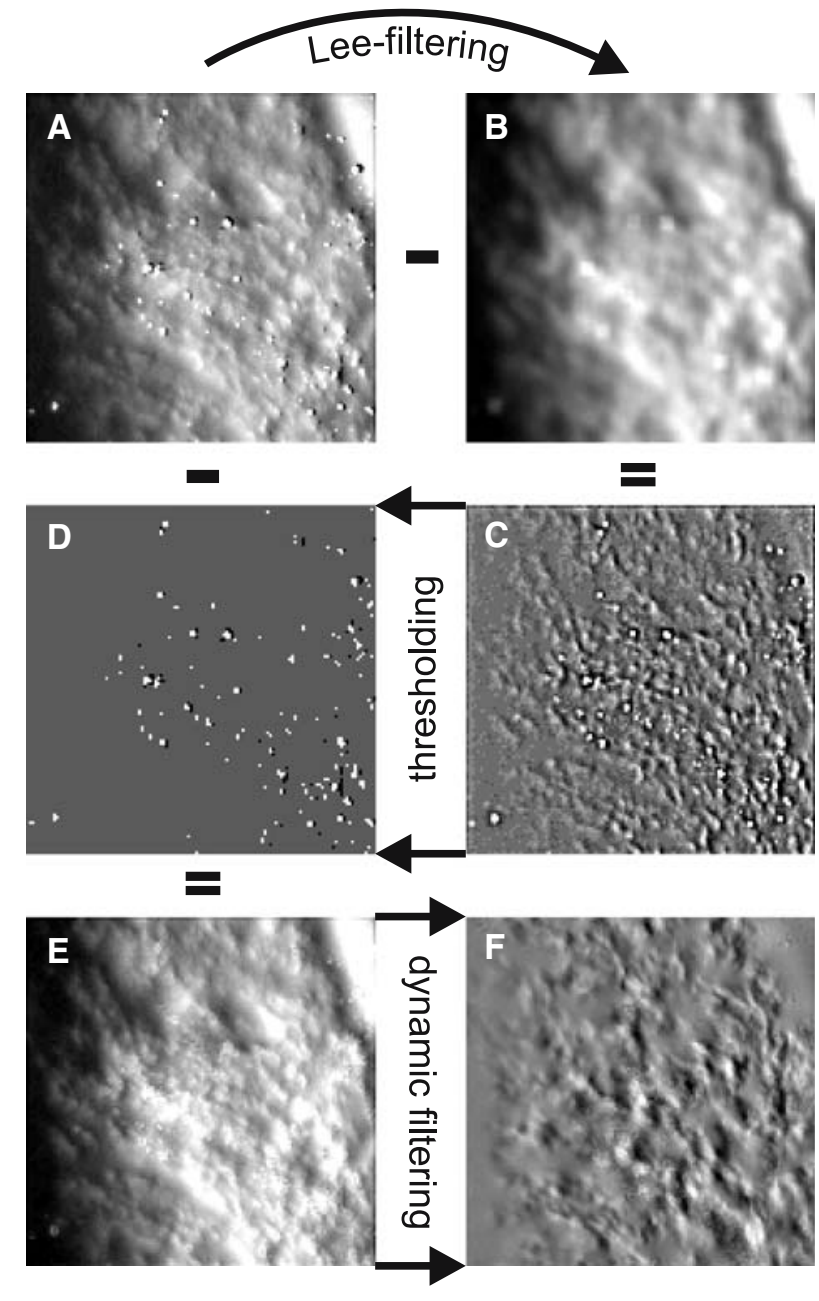

Fig. 5 Preprocessing. a Frame from a raw reflectometry movie. b Smoothed, Lee-filtered image. $\mathbf{c}=\mathbf{a}-\mathbf{b}$ tracers are enhanced, but there is still some residual background. $\mathbf{d}$ Tracers extracted by thresholding. $\mathbf{e}=\mathbf{a}-\mathbf{d}$ Tracers subtracted from the raw image. $\mathbf{f}$ Dynamic filtering yields "metachronal wave field"

The procedure is illustrated in Fig. 5: from the raw image (Fig. 5a) we subtract the smoothed, Lee-filtered image (Fig. 5b). The result (Fig. 5c) contains the tracers and some residual background, which is finally removed by thresholding: pixel values which deviate less than 2.5-times the standard deviation from mean are set to zero, yielding image in Fig. 5d. Such tracer movies will be subject of the analysis of mucociliary transport in Sect. "Mucociliary transport'.

Subtracting the tracers from the raw movie one obtains an intermediate Fig. 5e, which still contains the uninteresting features of the trachea topography. Since the topography features are static, they can be removed by "dynamic filtering", i.e., by filtering in the time domain: essentially we apply an appropriate band-pass filter to obtain the "metachronal wave field", as shown in Fig. 5f. (Note resulting contrast enhancement.) The design of the filter requires spectral analysis of the surface oscillations, as will be discussed in Sect. "Analyzing metachronal wave fields".

\section{Analyzing metachronal wave fields}

The characteristic features of the observed luminosity distribution $I(x, y, t)$ are the pronounced periodic oscillations in time and the wave-like appearance in space. It is therefore quite natural to analyze the data in terms of a superposition of two-dimensional harmonic waves:

$$
I(x, y, t)=\int_{\mathbf{k} \cdot f} \mathrm{~d} p \mathrm{~d} q \mathrm{~d} f A(\mathbf{k}, f) \cos (2 \pi f t-2 \pi \mathbf{k} \cdot r+\phi[\mathbf{k}, f])
$$

The temporal oscillation of a partial wave is characterized by the frequency $f=1 / T_{f}\left(\right.$ in $\mathrm{Hz}$ ), where $T_{f}$ is the oscillation period. The spatial oscillations are quantified by the wavevector $\mathbf{k}=(p, q)$, whose Cartesian components $p$ and $q$ are the spatial frequencies along $x$ and $y$ directions ${ }^{1}$. The wavelength is $\lambda=1 / k$, where $k=\sqrt{p^{2}+q^{2}}$ is the magnitude of the wave-vector. The wave front propagates with the velocity $c=f \lambda$ in the direction of $\mathbf{k}$. Note that this definition of the propagation direction implies that $f \geq 0$. Finally $A$ is the amplitude and $\varphi$ the phase shift of the partial wave with respect to the origin $x, y, t=0$. The motivation for the Fourier analysis is the hope for data reduction: one expects that only a narrow range of space and time frequencies is needed to characterize $I(x, y, t)$ resulting from well-coordinated ciliary beating.

The primary data set consists of discrete samples of the distribution $I(x, y, t)$ recorded in a finite interval $x \in\{0, X\}$, $y \in\{0, Y\}, t \in\{0, T\}$, with the resolutions $\Delta x, \Delta y, \Delta t$. Such data can be synthesized with a discrete and finite set of space and time frequencies $p, q$ and $f$. For the purpose of the computation we express this synthesis in complex notation:

$I[x, y, t]=\Re \sum_{-p_{N}}^{p_{N}} \sum_{-q_{N}}^{q_{N}} \sum_{f=0}^{f_{N}} F[p, q, f] \mathrm{e}^{i 2 \pi(f t / T-p x / X-q y / Y)}$

Here $F[p, q, f]=A[p, q, f] \exp (i \varphi[p, q, f])$ are the complex Fourier amplitudes of the partial waves, $\Re$ denotes the real part of a complex expression. In the summation limits $p_{N}=1 /(2 \Delta x), q_{N}=1 /(2 \Delta y)$ and $f_{N}=1 /(2 \Delta t)$ are the Nyquist frequencies, i.e., the maximal frequencies that can be detected in the sampled data. Note that whereas the

\footnotetext{
${ }^{1}$ To comply with the technical definition of frequency, we take the factor $2 \pi$ out of the definition of the wave-vector.
} 
spatial frequencies $p$ and $q$ can be positive or negative, the temporal frequencies $f$ are always positive. The amplitudes $F[p, q, f]$ are determined in the standard fashion by applying the three-dimensional discrete Fourier transform (DFT) to the data array $I[x, y, t]$. The DFT of the sampled distribution is defined as

$F\left[p^{\prime}, q^{\prime}, f^{\prime}\right]=\sum_{x=0}^{X-1} \sum_{y=0}^{Y-1} \sum_{t=0}^{T-1} I[x, y, t] \mathrm{e}^{-i 2 \pi\left(f^{\prime} t / T+p^{\prime} x / X+q^{\prime} y / Y\right)}$.

Note that for the purpose of computation we interpret $x=0, \ldots, X-1, y=0, \ldots, Y-1$ and $t=0, \ldots, T-1$ as the indices of a three-dimensional array $I[x, y, t]$ with $X \times Y \times T$ elements. The result of the DFT is the complex array $F\left[p^{\prime}, q^{\prime}, f^{\prime}\right]$ with indices $p^{\prime}, q^{\prime}$ and $f^{\prime}$ and with the same dimensions $X, Y$ and $T$. This array contains the desired Fourier amplitudes $F[p, q, f]$, but the proper identification of the array elements with the physical space and time frequencies is subtle because the $F\left[p^{\prime}, q^{\prime}, f^{\prime}\right]$-array contains redundant information. It turns out that the amplitudes of the positive (i.e., physical) time oscillation frequencies $f=0, \ldots, f_{N}$ are stored as follows: element $f^{\prime}=0$ contains the amplitude of $f=0$, but the amplitudes of the remaining ascending frequencies are in the array elements $f^{\prime}=T-1$, $T-2, \ldots, T / 2$ in descending order. This is in the so-called negative frequency range of the DFT array! This apparent confusion is the result of a conflict of the convention used in the physical definition of wave propagation (i.e., the minus sign of the wave-vector in Eq. 2) with the mathematical DFT conventions. We would not dwell on this technicality if we were not interested in the determination of the direction of the propagation of the metachronal waves: a wrong choice of the range would invert the direction of the $\mathbf{k}$-vectors.

The data processing was done using routines written in the Interactive Data Language IDL (RSI Inc.). This programming environment is designed for processing of large data arrays and contains many useful pre-defined routines. In particular, we made extensive use of IDL's efficient Fast Fourier Transform (FFT) algorithm, which is based on the Cooley-Tukey algorithm (James and John 1965).

\section{Measuring ciliary beat frequency $(\mathrm{CBF})$}

Averaged power spectrum: The first step of the data analysis is a straightforward estimation of the power spectrum of the ciliary oscillations. (In general, the power spectrum of a time series $I[t]$ is defined as the complex square of the Fourier amplitudes: $P[f]=|F[f]|^{2}=A[f]^{2}$. Note that the information about the phase $\varphi$ is lost.) First we determine the area averaged power spectrum $\bar{P}[f]$ :
$\bar{P}[f]=\frac{1}{X Y} \sum_{x, y}\left|F_{x, y}[f]\right|^{2}=\frac{1}{X Y} \sum_{p, q}|F[p, q, f]|^{2}$.

Here $F_{x, y}[f]$ is the one-dimensional DFT of the time-series $I_{x, y}[t]$ at the position $[\mathrm{x}, \mathrm{y}]$ of the data array. In other words, $\bar{P}[f]$ can be obtained calculating the power spectra $P_{x, y}[f]=\left|F_{x, y}[f]\right|^{2}$ of individual pixels and subsequently averaging the results. In the second part of Eq. 5 we recall Parseval's theorem, which suggests an alternative scheme for the calculation of $\bar{P}[f]$.

A typical area averaged power spectrum $\bar{P}[f]$ (see Fig. 6) exhibits a pronounced peak centered at the fundamental frequency $f_{f}$ as well as a series of peaks at $2 f_{f}, 3 f_{f}, \ldots$ The presence of the higher order harmonics indicates that the oscillations of the sample surface are periodic but not purely sinusoidal. This is fortunate, recalling that the reflection contrast responds to the square of the slope of the surface: with purely sinusoidal surface oscillations we would pick up twice the frequency of the surface oscillation (recall that $2 \sin (\omega t)^{2}=1-\cos (2 \omega t)$ ). In order to make sure that we are indeed not picking-up any artifacts associated with our particular contrast technique, we compared the reflectometry data on $\mathrm{CBF}$ with data obtained by an independent technique, namely dynamic laser light scattering (Wessel et al. 1999). A comparison of CBF values as a function of temperature, as shown in Fig. 7, verified the equivalence of both techniques.

Thus, we interpret the fundamental frequency $f_{f}$ as the ciliary beat frequency CBF. For its accurate determination we cut out the fundamental peak, as marked by the shaded strip in Fig. 6, and estimate $f_{f} \equiv \mathrm{CBF}$ as the first central moment of the distribution. At the same time we also estimate the variance $\sigma_{f}^{2}$. Typical CBF values at $30^{\circ} \mathrm{C}$ are between 10 and $15 \mathrm{~Hz}$, typical standard deviations 1$4 \mathrm{~Hz}$.

Activity map: Having gained an averaged view of the spectrum of the surface oscillations, we turn to a positionresolved analysis of the ciliary activity. Figure 8 shows two

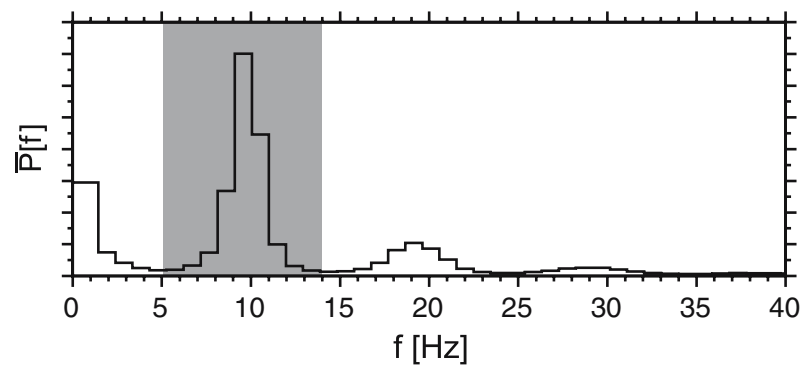

Fig. 6 A typical area averaged power spectrum. The fundamental peak indicates the $\mathrm{CBF}$, the number of harmonics varies from sample to sample. The resolution of $1 \mathrm{~Hz}$ corresponds to a recording time of $1 \mathrm{~s}$ 


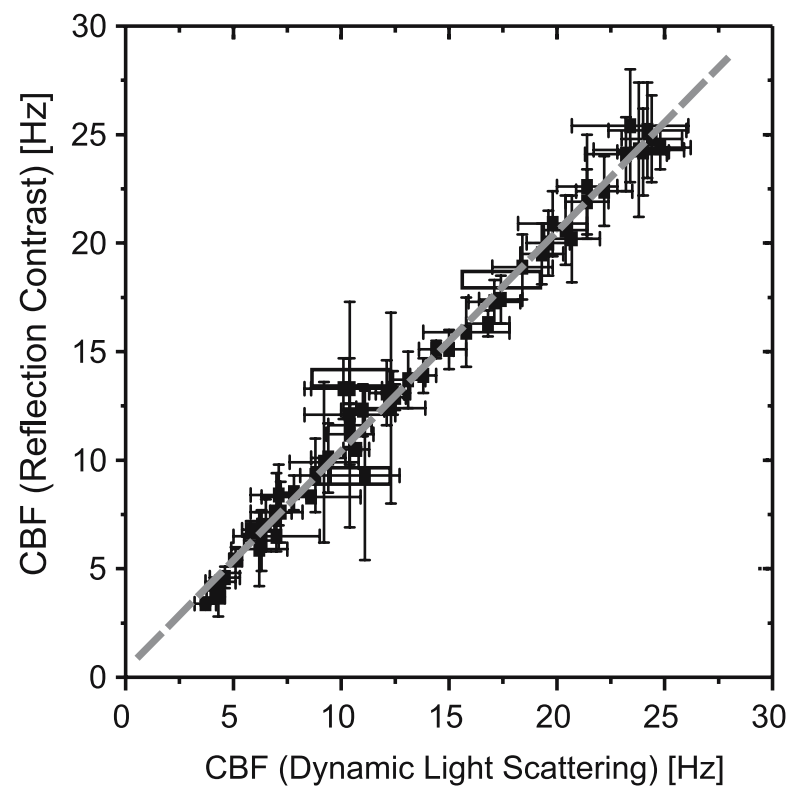

Fig. 7 Comparison of the $\mathrm{CBF}$ obtained by reflectometry and dynamic speckle interferometry. We varied the beat frequency by varying the temperature of the sample in the range between 22 and $42^{\circ} \mathrm{C}$. Error bars denote the width of the fundamental peak
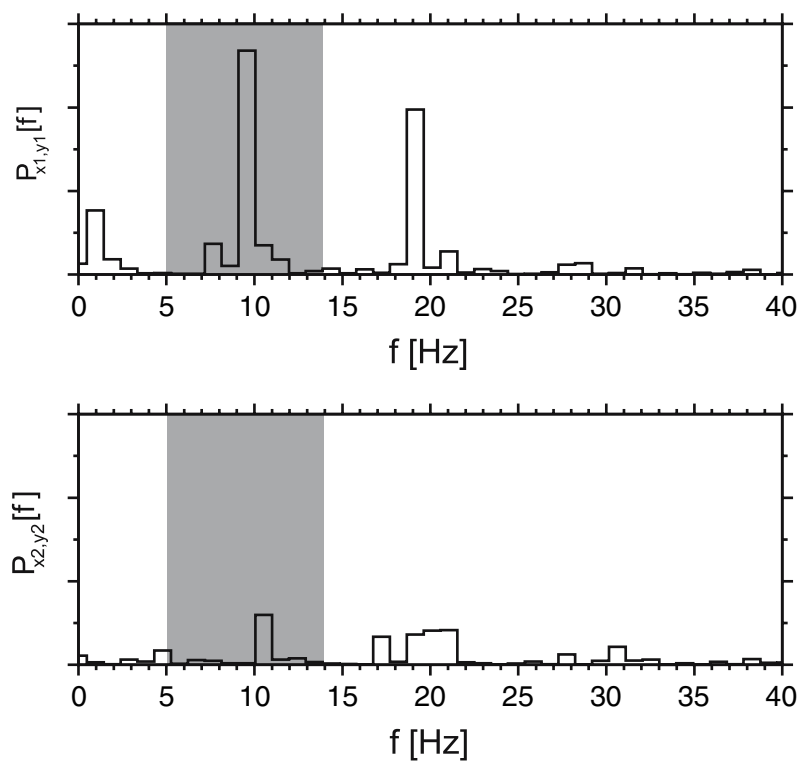

Fig. 8 Two examples of power spectra of time series from individual pixels. The shaded strip indicates the width of the fundamental peak of the area averaged spectrum

examples of power spectra of time series from two individual pixels. Unlike the area-averaged power spectrum, the individual spectra tend to be rather noisy. Sometimes one finds regions with low (visible) ciliary activity, with a poorly defined fundamental peak (lower graph of Fig. 8).
To discriminate such ill-defined data we exploit the information obtained from the average. For each pixel $x, y$ we measure the integral $A_{x, y}$ of the power spectrum in a frequency band corresponding to the width of the averaged fundamental peak. A reasonable choice is $f_{f} \pm 2.6 \sigma_{f}$ that would cover $99 \%$ of a normal distribution. Then we compare $A_{x, y}$ with the area $\bar{A}$ of the averaged fundamental peak (shaded strip in Fig. 8). If the ratio $A_{x, y} / \bar{A}$ is smaller than a chosen value of 0.15 , then we mark the pixel as invalid (white in Fig. 9). To estimate the CBF in a valid pixel we determine the mean frequency $f_{x, y}$ in the $f_{f} \pm 2.6 \sigma_{f}$-range. From these local estimates $f_{x, y}$ we construct the activity map (see Fig. 9). Note that given the $2.2 \mu \mathrm{m}$ spatial resolution of our setup, we are able to determine the CBF of a single ciliated cell. The representative example in Fig. 9 exhibits a smooth spatial variation of $\mathrm{CBF}$, as indicated in the histogram (see Fig. 10). As we shall see later, the local frequency in an area of the epithelium encompassing a few ciliated cells is remarkably stable in time over many oscillation periods.
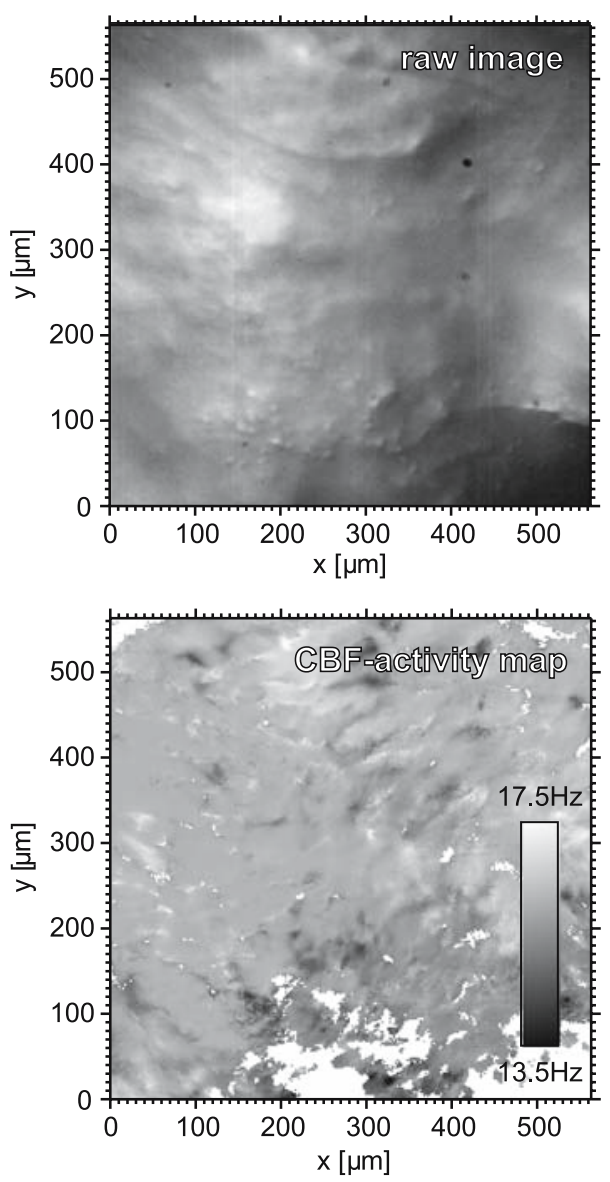

Fig. 9 Typical image of a sheep trachea and the resulting CBFactivity map. White patches indicate an ill-defined fundamental peak i.e. regions with hardly visible ciliary activity 


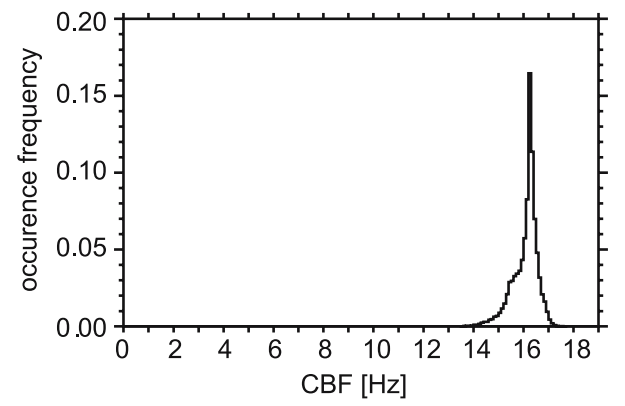

Fig. 10 Histogram of CBF-values from the activity map in Fig. 9

Metachronal waves

Using the information on the power spectrum of the ciliary beat we are now in the position to extract the "metachronal wave field", as indicated in Sect. "Analyzing metachronal wave fields". To the raw image from Fig. 9 we apply a filter, selecting only the frequency components $f$ which represent the ciliary oscillation, i.e., narrow frequency bands around the the fundamental CBF peak and of all detectable harmonics. A typical result is shown in Fig. 11, see also movies $\mathrm{S} 1$ and $\mathrm{S} 2$ in the supplementary material.
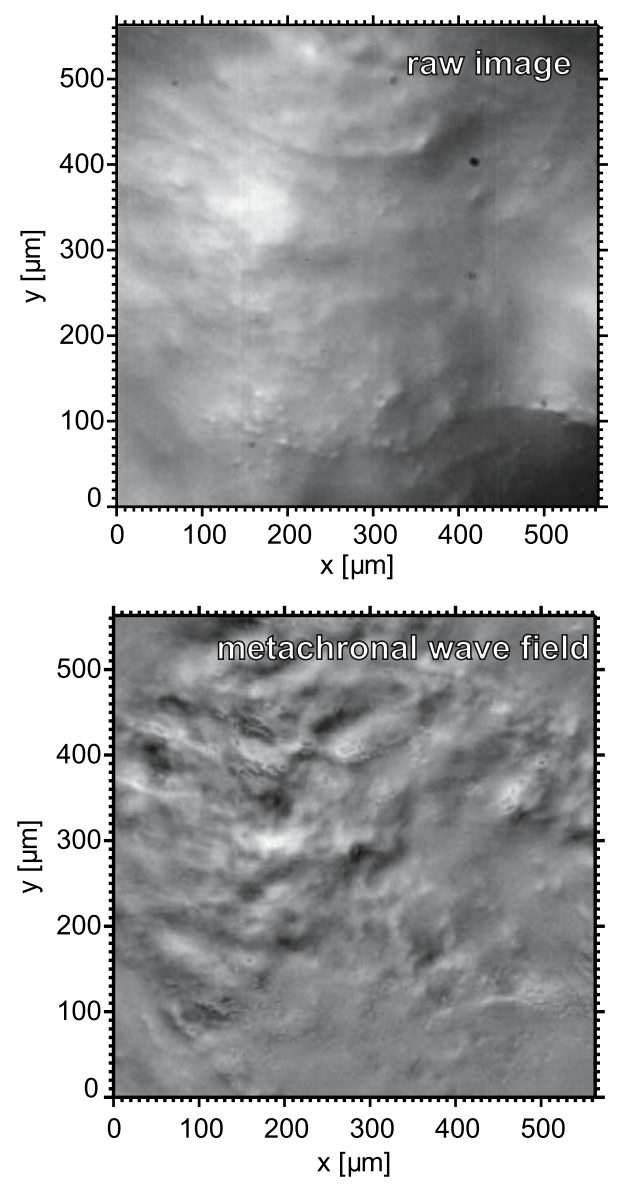

Fig. 11 Raw image and extracted metachronal wave field
Spatial structure of the wave field

In analogy with the analysis of temporal oscillations, the spatial structure of the wave field can be characterized in terms of the average two-dimensional power spectrum $\bar{P}_{\mathbf{k}}[p, q]$ of the spatial frequencies $p, q$. However, unlike the time-frequency case, $\bar{P}_{\mathbf{k}}$ cannot be calculated by averaging the spatial power spectra of the individual pictures over time, because the two-dimensional Fourier spectra of real data sets are symmetrical with respect to the origin. Thus, the direction of wave propagation would be lost. Fortunately this ambiguous symmetry can be removed in the frequency domain: We employ Parseval's theorem (recall second part of Eq. 5), but in order to preserve the propagation direction, we only sum over the physical frequencies $f$ from the three-dimensional FFT array $F[p, q, f]$, as discussed in the introduction to Sect. "Analyzing metachronal wave fields". Thus, we define the spatial power spectrum as:

$\bar{P}_{\mathbf{k}}[p, q]=\sum_{f=0}^{f_{N}}|F[p, q, f]|^{2}$.

The power spectrum $\bar{P}_{\mathbf{k}}$ represents the distribution of the wave-vectors $\mathbf{k}$ of the partial waves. A typical example is shown in Fig. 12a. Usually the distribution exhibits two distinct clouds, whose centroids define two main wave vectors, marked in Fig. 12 by the two arrows. The main wave vector corresponding to the larger peak is oriented more or less upward, i.e., in the direction of pharynx and thus in the direction of the mucociliary transport. The smaller peak points downwards in the opposite direction. However, the upward component dominates, a typical ratio of the powers under the two peaks is 2:1. These findings suggest that the mucus transport follows the direction of the waves, but the picture is not always as clear-cut as suggested by the distribution in Fig. 12. For example, quite often one finds multiple clouds of wavevectors $\mathbf{k}$ making it difficult to estimate the direction of the wave motion. In the next sections we shall investigate alternative techniques to refine and complement the analysis.

To conclude the spatial analysis, we note that the distribution $\bar{P}_{\mathbf{k}}[\mathbf{k}]$ can be used to estimate a typical wavelength of the metachronal wave field. For this estimation we determine the histogram $P[k] \Delta k$ of the distribution of the absolute values $k=|\mathbf{k}|=1 / \lambda$. A value in the histogram is obtained by integrating $\bar{P}_{\mathbf{k}}[\mathbf{k}]$ over an annulus with radius $k$ and thickness $\Delta k$. A typical result is plotted in Fig. 12b. We note that the $P[k]$-distribution can be well approximated by a log-normal distribution, the dashed line in Fig. 12b. The log-normal distribution function $f(k)=\exp \left[-(\ln k-\mu)^{2} /\right.$ $\left.2 \sigma^{2}\right] /(\sqrt{2 \pi} \sigma k)$ exhibits a convenient property: the modus 

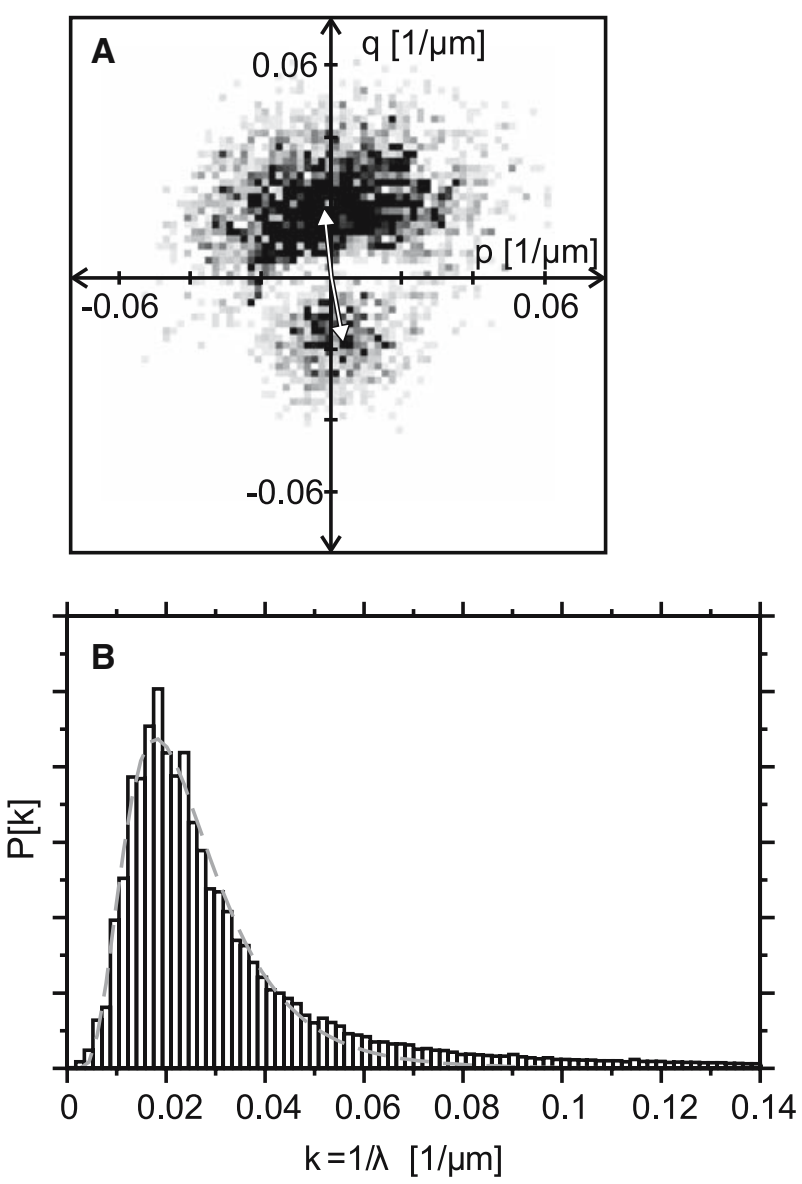

Fig. 12 Spatial structure of the wave field. a Power spectrum, b Histogram of the absolute value $\mid \mathrm{kl}$. Dashed line is the best fit by lognormal distribution

of $f(k)$ equals the mean of $1 / k$. This property provides a robust estimate of the mean wavelength as $\bar{\lambda}=1 / k_{\mathrm{m}}$, where $k_{\mathrm{m}}$ is the position of the modus of the log-normal fit. The measured values of $\bar{\lambda}$ are found in a relatively broad range of $40-100 \mu \mathrm{m}$. Combining these values with the typical oscillation frequencies $f_{f}$ between 10 and $15 \mathrm{~Hz}$, one may expect wave-velocities $\bar{\lambda} f_{f}$ in the order of $1,000 \mu \mathrm{m} / \mathrm{s}$. However, considering the fact that our waves are by no means purely harmonic, such combination of spatial and temporal features is rather crude and must be taken with caution.

\section{Space-time correlations}

Because of its three-dimensional nature and apparent randomness, the analysis of the "metachronal wave field" is not an easy task. Therefore we have so far analyzed the temporal and spatial features separately, in terms of the averaged temporal power spectrum $\bar{P}[f]$ and of the wavevector distribution $\bar{P}_{\mathbf{k}}[\mathbf{k}]$. This approach provided a first overview, but now we would like to combine space and time. This could be done by examining the three-dimensional power spectrum $P[p, q, f]=|F[p, q, f]|^{2}$. However, the interpretation of $P[\mathbf{k}, f]$ is difficult because of the broad distribution of the $\mathbf{k}$-vectors. As the hope for data reduction in the k-domain failed, we return to the real space. We investigate the three-dimensional space-time correlation function $C[\xi, v, \tau]$. In fact, the correlation function $C[\xi, v, \tau]$ and the power spectrum $P[p, q, f]$ are a pair of Fourier transforms (the well known Wiener-Khinchin-Theorem; Press et al. 1992) and therefore contain the same information. However, certain features of the wave field are more easily discerned in $C[\xi, v, \tau]$ than in $P[p, q, f]$. In real space $C[\xi, v, \tau]$ is defined as

$C[\xi, v, \tau]=\sum_{x, y, t} I[x, y, t] I[x+\xi, y+v, t+\tau]$.

To obtain $C[\xi, v, \tau]$ at some $\xi, v, \tau$, one multiplies pairs of images, displaced by $\xi, v$ and separated by a time lag $\tau$ and adds up the results for all $x, y, t$. The result is a new image stack in real space, but instead of $x, y$ and $t$, the coordinates are now the displacements $\xi, v$ and the time lag $\tau$, as indicated in Fig. 13. The direct calculation of the space-time correlation in real space is rather time consuming and therefore we exploit the Wiener-Khinchin-Theorem: FFT provides the most efficient numerical algorithm for the calculation of $C[\xi, v, \tau]$. However, since we expect the time correlation to persist over a period of time comparable with the measurement period $T$, we must take precautions to avoid the wrap-around artifact associated with the periodic nature of the FFT: before calculating $F[p, q, f]$ we apply the zero padding (Press et al. 1992) on the time axis.

The best insight into the nature of the space-time correlation can be gained when viewing the image stack $C[\xi, v, \tau]$, an example is the movie S3 in the supplementary material. Figure 13, that shows a few frames of the movie, is a barely adequate substitute. A first impression from a correlation movie are vivid oscillations in the central region, whose amplitude rapidly diminishes with increasing $\tau$. The time correlation profile $C_{0,0}[\tau](C[\xi, v, \tau]$ at $\xi=0, v=0)$

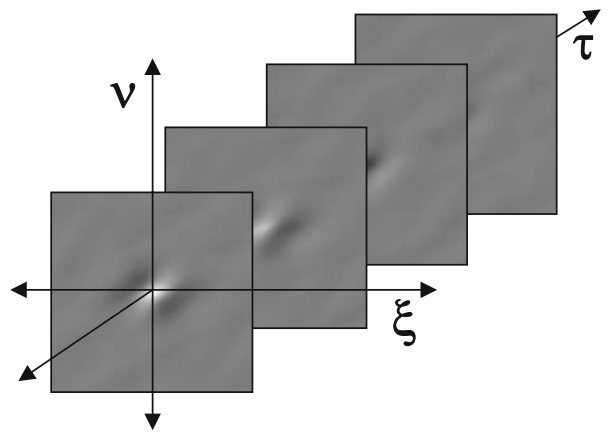

Fig. 13 Space-time correlations 
is the DFT of the area averaged power spectrum $\bar{P}[f]$. An example is shown in Fig. 14. The correlation function oscillates with the CBF and decorrelates within a few oscillation periods. Since $C_{0,0}[\tau]$ and $\bar{P}[f]$ are a pair of Fourier transforms, the de-correlation time can be defined as $\tau_{c}=1 / \sigma_{\mathrm{f}}$, where $\sigma_{\mathrm{f}}$ is the width of the fundamental peak. To understand the reason for the rapid de-correlation, we examine the auto-correlation functions $C_{x, y}[\tau]=\sum_{t} I_{x, y}[t]$ $I_{x, y}[t+\tau]$ of the time series $I_{x, y}[t]$ from the individual pixels. (Recall that one pixel corresponds to a fraction of a ciliated cell.) Two examples of pixel-autocorrelation functions are shown in Fig. 15. In contrast to the overall autocorrelation $C_{0,0}[\tau]$ in Fig. 14, there is hardly any decrease of the oscillation amplitude in Fig. 15a. Except for slow dephasing, the beat remains remarkably stable over many oscillation periods. However, occasionally one observes nodes indicated by arrows in Fig. 15b. These nodes indicate phase jumps in the otherwise periodic time series $I_{x, y}[t]$. Such phase jumps are the main reason for the rapid decorrelation of the overall autocorrelation $C[0,0, \tau]$, which is simply the sum of the individual pixel autocorrelations $C_{x, y}[\tau]$. We shall return to the phase jump phenomenon later in the next section.

For now we return to the $C[\xi, v, \tau]$-movie and take a closer look at the individual frames. The example in Fig. 16 is the frame $C[\xi, v, 0]$, representing the spatial

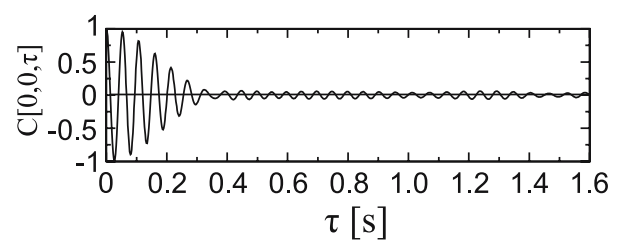

Fig. 14 Typical time correlation function $C_{0,0}[\tau]$ of the temporal information from all pixels in an image sequence (area of $0.25 \mathrm{~mm}^{2}$ ). Typically a de-correlation is observed (here after about $0.3 \mathrm{~s}$ )
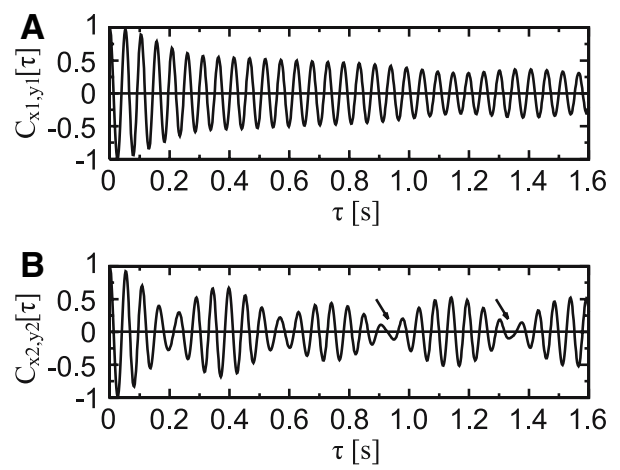

Fig. 15 Two typical auto-correlation functions of the time series from two individual pixels (area of about $13 \mu \mathrm{m}^{2}$ ). De-correlation due to a slow dephasing is observed. Phase jumps in the time correlation function are indicated by arrows auto-correlation at $\tau=0$. We observe a slightly elongated spatial correlation pattern, consisting of a central extremum accompanied by a couple of side fringes along the broader side of the pattern. Obviously, the fringes are the remainants of the spatial periodicity of the wave field. One can regard the correlogram as a representation of the "mean wave". The correlation profile along the line in Fig. 16 is plotted with a solid line in Fig. 17. Unlike the temporal correlation, the spatial correlation is limited to only about one wavelength. Note that the spacing of the spatial correlation minima provides an independent estimate of $\lambda$, as indicated in Fig. 17. The estimates from the correlation minima are about $20 \%$ smaller than those from the wavevector distribution. This is a very good agreement, considering that the two estimates are two rather different averages of a broad distribution.

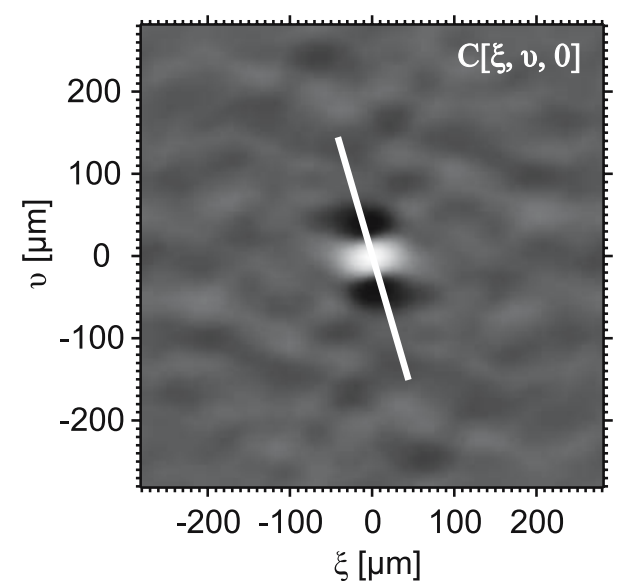

Fig. 16 Spatial auto-correlogram representating the mean wave. The spatial oscillations indicate a certain degree of spatial periodicity in the metachronal wave data $I[x, y, t]$

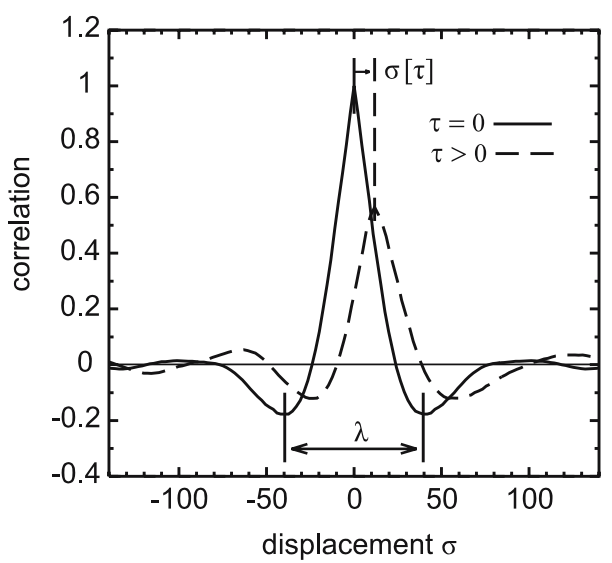

Fig. 17 Correlogram line profiles, along the white line in Fig. 16, at two different time lags. $\tau=0$ is the spatial-autocorrelation and $\tau>0$ the space-time cross-correlation. The gradual shift $\sigma[\tau]$ of the maximum allows the determination of the velocity of the mean wave 
Now we put the $C[\xi, v, \tau]$-movie in slow motion and observe the changes of the correlation pattern. With increasing time-lag $\tau$ the extremum oscillates between positive and negative values until it de-correlates as discussed above. However, before the correlation is lost, the extremum also shifts its position in a direction that is nearly perpendicular to the broad side of the pattern. This is illustrated in Fig. 17, where we plot the profile along the white line in Fig. 16 for two time-lags $\tau=0 \mathrm{~ms}$ and $\tau=8 \mathrm{~ms}$, corresponding to about $1 / 10$ of the beat period $T_{f}$. The shift of the correlation peak is an unambiguous signature of the wave propagation and can be used to estimate a mean wave velocity, namely the velocity of the mean wave. We measure the vectorial displacement $\sigma=\left(\sigma_{x}, \sigma_{y}\right)$ of the correlation maximum with respect to the origin $\xi=0, v=0$ for a series of time-lags $\tau$ and then we determine the velocity $\mathbf{v}_{\text {wave }}$ of the mean wave as

$\mathbf{v}_{\mathrm{wave}}=\lim _{\tau \rightarrow 0} \frac{\sigma[\tau]}{\tau}$.

Typical metachronal wave speeds determined by this technique are between $100-400 \mu \mathrm{m} / \mathrm{s}$. The propagation angles are between $-16^{\circ}$ and $40^{\circ}$ with respect to upward direction, i.e., wave propagation is directed essentially towards the pharynx.

\section{Phase synchronization}

The term "metachronal coordination"' means that the cilia maintain a well defined relation of their beating phases over a more or less large area of the trachea epithelium. Thus, the most direct view on the coordination phenomena can be obtained by analyzing the space distribution of the phase of the surface oscillations. In principle, all necessary phase information is contained in the complex Fourier amplitudes $F=A \exp (i \varphi)$; so far we neglected $\varphi$, concentrating on the power spectra $P=|F|^{2}=A^{2}$.

In designing the procedure for the phase extraction we were guided by the following observations: From the area averaged power spectrum $\bar{P}[f]$ and from the activity map we know that the distribution of the oscillation frequency on the trachea surface is smooth, with a standard deviation $\sigma_{f}$ of about $1 / 10$ of the fundamental frequency $f_{f}$. From the time correlation data we expect that the time series $I_{x, y}[t]$ from a single pixel is remarkably stationary over a number of beat periods; we only observe slow dephasing interrupted by occasional phase jumps. Regarding these properties we are in the position to determine both, the position- as well as the time-dependence of the phase of the ciliary oscillation. The concept of the so called short-time Fourier transform (STFT), also named time-dependent or sliding Fourier transform, provides the necessary tool

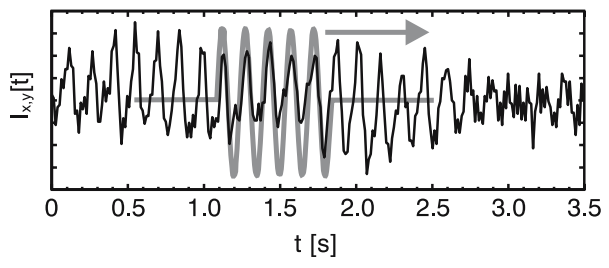

Fig. 18 Measuring the phase. The times series $I_{x, y}[t]$ from a pixel is cross-correlated with a windowed complex harmonic. Sliding the window over $I_{x, y}[t]$ we determine the time-dependence of the phase $\Phi_{x, y}[t]$

(Jacobsen and Lyons 2003). The idea is to determine the desired complex Fourier amplitude $F_{f}[t]=A[t] \exp (i \varphi[t])$ of the fundamental frequency $f_{f}$ in a window that is slid across the time series $I_{x, y}[t]$ :

$F_{f}[t]=\sum_{s=0}^{W-1} I_{x, y}[t+s] w[s] \mathrm{e}^{-i 2 \pi f_{f} s / T}$

The procedure is illustrated in Fig. 18. Note that the operation is equivalent to cross-correlating the time-series $I_{x, y}[t]$ with a windowed complex harmonic. The window $w[s]$ is an array of length $W<T$. The simplest choice is a rectangular window, that is $w[s]=1 \forall s$, but more sophisticated window shapes, such as the popular Hamming window $w[s]=\alpha+(1-\alpha) \cos [\pi s / W], \quad \alpha=0.54$, can be considered to optimize the procedure. More important than the exact shape of the window is a proper choice of the width $W$ of the window, because the width determines the frequency resolution. A rectangle extracts approximately the frequency range $\Delta f=1 / W \Delta t$. We wish to select just the frequencies under the fundamental peak of the area averaged power spectra and therefore we set $W \approx 1 / 2 \sigma_{f} \Delta t$. The result of the sliding FT of $I_{x, y}[t]$ is a complex array $F_{f}[t]=A_{f}[t] \exp \left(i \Phi_{f}[t]\right)$. This time we discard the modulae $A_{f}[t]$ and extract the phases according to $\Phi_{f}[t]=$ $\arg \left(F_{f}[t]\right)=\arctan \left(\Im F_{f} / \Re F_{f}\right)$. We observe an essentially linear evolution of the phase, which is wrapped into a saw-tooth curve (see Fig. 19), since phase can only be determined $\bmod 2 \pi$. This smooth linear phase evolution confirms a previous result from the analysis of the time correlation: The phase is remarkably stable over many oscillation periods $T_{f}$. Occasionally, however, one encounters non-trivial phase jumps as indicated by the arrow in Fig. 19.

Applying the STFT operation to all pixels, we obtain a complex space-time array

$F_{f}[x, y, t]=A_{f}[x, y, t] \exp \left(i \Phi_{f}[x, y, t]\right)$,

from which we extract the phases $\Phi_{f}[x, y, t]$. This new image stack provides a new kind of movie of the surface 


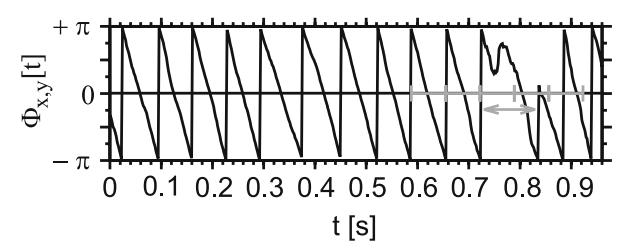

Fig. 19 Time evolution of the phase in a representative pixel from Fig. 21 (phasemap), but occasionally we observe jumps indicated by the arrow

modulation: we call this a dynamic phase contrast movie. An example is provided in the supplementary material, movie S4. In Figs. 20 and 21 we show two examples of single frames out of such sequences.

The first impression from such a movie is a pattern of smooth looking gradually shaded patches, interrupted only by sharp black-white borders indicating the $2 \pi$ wraparound. In the course of the movie one can watch the black-white borders moving in the direction of the wave propagation. The observation can be interpreted as follows:
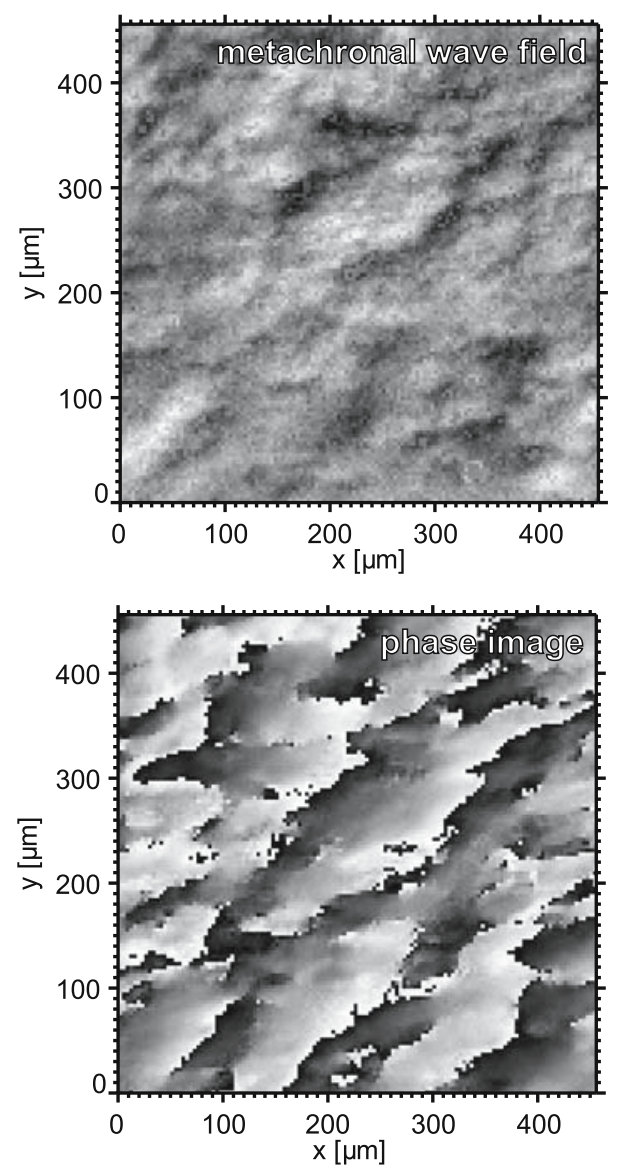

Fig. 20 Example (bovine trachea) of wave field images recorded with the Dalsa CA-D1 camera $(128 \times 128$ pixels $)$. The phase image exhibits exceptionally well developed metachronism, i.e. long wavelength
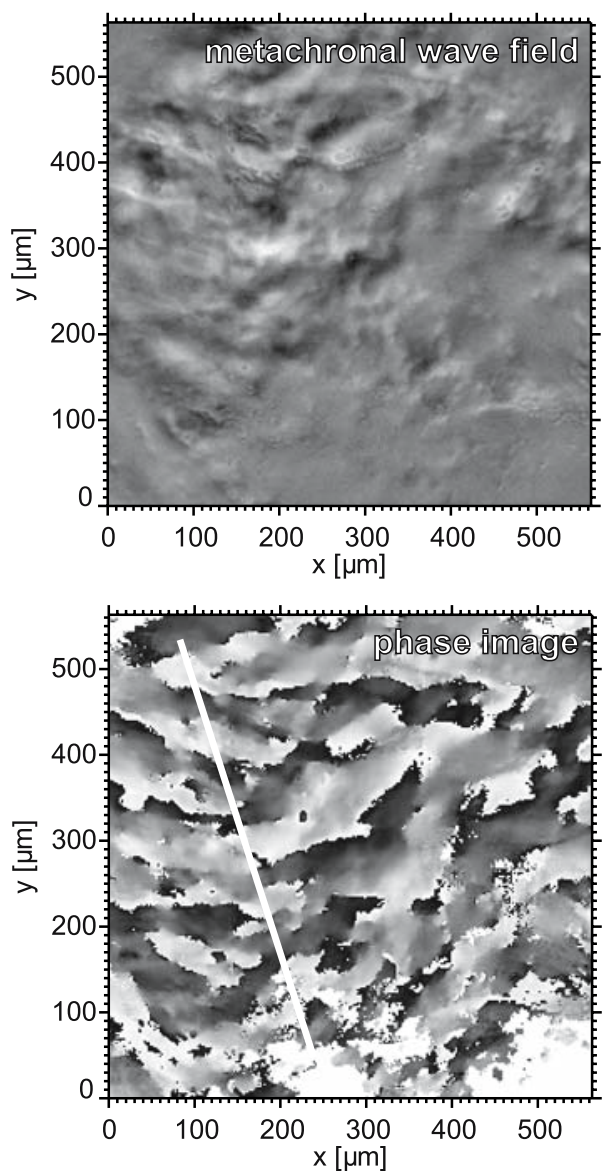

Fig. 21 Example (sheep trachea) of wave field images recorded with the Dalsa CA-D6 camera $(256 \times 256$ pixels $)$ is more typical: the white colored patches in the lower right corner of the phase image mark regions with low ciliary activity (recall Fig. 8). White line indicates the line profile shown in Fig. 23

In such smooth areas the cilia are well coordinated. Within the coordination patch there is a well defined frequency $f_{f}$ and well defined wave-vector $\mathbf{k}_{f}$. Therefore, in such areas the phase evolves essentially linearly in time and space according to:

$\Phi_{f}[x, y, t]=2 \pi f_{f} t-2 \pi \mathbf{k}_{f} \cdot \mathbf{r}+\varphi[x, y, t]$,

where $\phi[x, y, t]$ is a smooth, slowly varying function of $x, y, t$. In other words, locally the variation of the luminosity can be modeled as $I[x, y, t] \propto \cos \left(2 \pi f_{f} t+2 \pi \mathbf{k}_{f} \mathbf{r}+\phi[x, y, t]\right)$ (recall that the higher harmonics are now filtered out). However, upon a closer examination one finds that the smooth patches are occasionally interrupted by phase jumps of about $1 \pi$. Figure 22 is an attempt to emphasize these features in a pseudo- $3 \mathrm{~d}$ representation of a frame from the phase image in Fig. 20. Perhaps easier to understand are the line profiles in Fig. 23, where we have removed the $2 \pi$-wraparounds in order to improve the readability. 


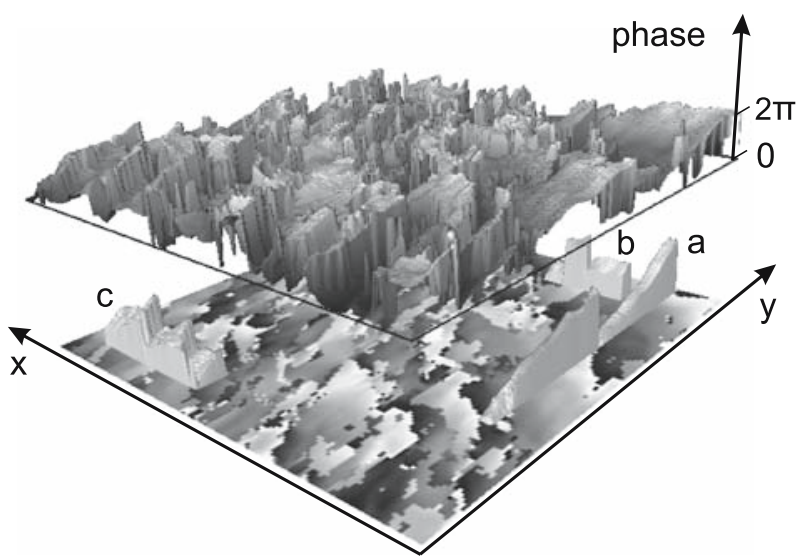

Fig. 22 An attempt for 3D representation of the phase image from Fig. 20. The detail $a$ indicates an area of continuously evolving phase whereas the areas $b$ and $c$ show neighboring regions of $\pi$ shifted beating phase

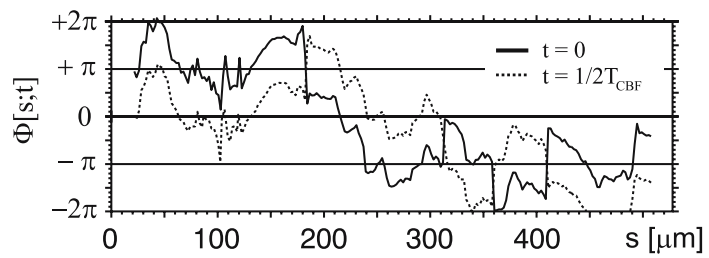

Fig. 23 Reconstruction of line profiles $\Phi_{f}[s ; t]$ taken along the white line $s$ in the phase image shown in Fig. 21 at two times $t$. The dark solid line and the dashed line are separated by $1 / 2$ of the oscillation period $T_{\mathrm{CBF}}$

The two curves represent line profiles $\Phi_{f}[s ; t]$ taken along the same line $s$ at two different times $t$ separated by $1 / 2$ of the $\mathrm{CBF}$ oscillation period $T_{\mathrm{CBF}}$. A particularly interesting feature of the line profiles $\Phi_{f}[s ; t]$ are occasional phase jumps by about $1 \pi$, which we already observed in Fig. 22. Such phase jumps occur in all analyzed metachronal wave fields and seem to be a significant feature of the metachronal coordination. Therefore we designed a procedure for a detailed investigation of the phenomenon.

The quantity in which we are interested is the gradient $\mathbf{G}[x, y]=\nabla \Phi_{f}[x, y]=\left(\partial \Phi_{f} / \partial x, \partial \Phi_{f} / \partial y\right)$ of the two dimensional phase distribution. Estimation of differential quantities from realistic, i.e., noise affected, data always involves a trade-off between position resolution and noisiness. Fortunately the quality of the phase data is sufficient to preserve a position resolution of one pixel employing a simple 4 pixel estimator similar to the Roberts cross edge detector (Roberts 1965). We estimate the components of the gradient vector $\mathbf{G}[x, y, t]$ as

$$
\begin{aligned}
G_{x}[x, y, t] & =\frac{1}{2 \Delta x}\left(\Delta \phi_{r}[x, y, t]-\Delta \phi_{l}[x, y, t]\right) \\
G_{y}[x, y, t] & =\frac{1}{2 \Delta y}\left(\Delta \phi_{r}[x, y, t]+\Delta \phi_{l}[x, y, t]\right),
\end{aligned}
$$

where

$$
\begin{aligned}
\Delta \phi_{r}[x, y] & =\Phi[x+1, y+1]-\Phi[x, y] \\
& =\arg \left(F_{f}[x+1, y+1] F_{f}^{*}[x, y]\right) \\
\Delta \phi_{l}[x, y] & =\Phi[x, y+1]-\Phi[x+1, y] \\
& =\arg \left(F_{f}[x, y+1] F_{f}^{*}[x+1, y]\right) .
\end{aligned}
$$

Note here a subtlety: we do not estimate the phase differences from a frame $\Phi_{f}[x, y]$ of the phase movie, because this would emphasize the artificial $2 \pi$-phase jumps. Instead we return to the complex results of the sliding FT, Eq. 10 and use the identity

$A[\mathbf{r}] \exp (i \Phi[\mathbf{r}]) A\left[\mathbf{r}^{\prime}\right] \exp \left(-i \Phi\left[\mathbf{r}^{\prime}\right]\right)=A[\mathbf{r}] A\left[\mathbf{r}^{\prime}\right] \exp (-i \Delta \Phi)$.

At first glance this identity appears trivial, but using its right hand version we get rid of the phase wrap artifact because the phase of the complex product $F_{f}[x, y] F_{f}^{*}\left[x^{\prime}, y^{\prime}\right]$ never exceeds $2 \pi$, provided that the points $x, y$ and $x^{\prime}, y^{\prime}$ are sufficiently close to each other.

The gradient operation yields two movies for the two cartesian components of the vector $\mathbf{G}[x, y, t]$. However, we prefer to work in polar coordinates, transforming the data into the modulus $|\mathbf{G}[x, y, t]|$ and the direction angle $\arg \mathbf{G}[x, y, t]$. The distribution of the modulus $|\mathbf{G}[x, y, t]|$ in a frame of the gradient movie is shown in Figs. 24 and 25 (watch also the movie S5 in the supplementary material). In both examples we observe a web of white lines subdividing the picture. The white lines represent edges with a magnitude close to $\pi$. The location of the $\pi$-jumps is quite stationary: they persist over several oscillation periods, as documented in Fig. 26.

Apparently, these phase jumps are organized in "defect lines" surrounding more or less smooth patches of gradual phase evolution. The shape of the patches cannot be easily defined, but they appear slightly elongated, about $50 \mu \mathrm{m}$ wide on the shorter side. (Note the strong resemblance of the pattern with the electro-micrograph of rabbit tracheal epithelium shown in Fig. 1 of Ref. Sanderson and Sleigh 1981). The width of the patches corresponds roughly to the typical metachronal wavelength, as it was derived from the spatial analysis of the wave field. Thus, the spatial correlation function (see Fig. 16) appears to reflect the average of the decorrelation due to the defect lines. Within the smooth patches, between the $\pi$ defect lines, we expect a gradual phase evolution according to Eq. 11 . Thus, the quantity $\mathbf{k}[x, y]=-\mathbf{G}[x, y] / 2 \pi$ can be interpreted as the spatial distribution of the wave vectors $\mathbf{k}$. (Likewise the quantity $V[x, y]=-2 \pi f_{f} /|\mathbf{G}[x, y]|$ can be interpreted as the spatial distribution of the phase velocity). This interpretation is confirmed in Fig. 27: the histogram of the 

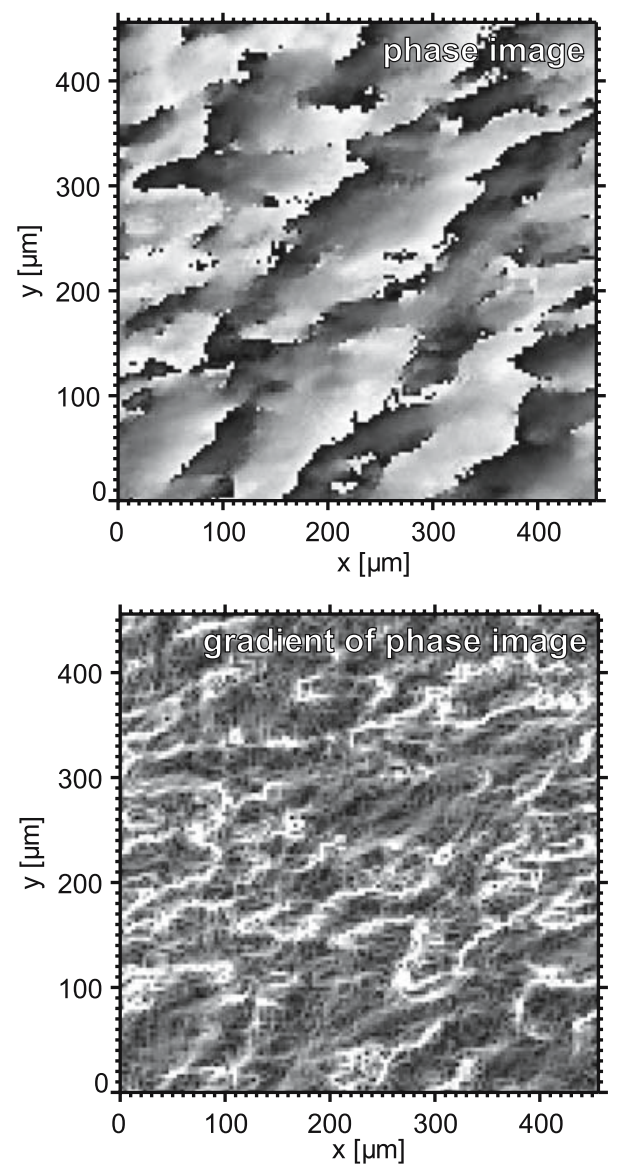

Fig. 24 Gradienting the phase data from a bovine trachea. Lower part shows the modulus of the gradient, i.e., $|\mathbf{G}[x, y, t]|$. The contrast is slightly enhanced by $\gamma=0.7$ to show the defect lines corresponding to $\pi$-jumps together with the background within coordinated patches

modulus $|\mathbf{k}[x, y]|$ agrees well with the histogram of $\mathbf{k}$ found in Fig. 12. This is a significant finding: recall that in Fig. 12 we analyzed the spatial structure of the wave field, whereas now we are looking at the phase of the oscillations in time. The consistency of these complementary space and time views of the metachronal coordination is further confirmed in Fig. 28a where we show an example of the distribution of the angle of the wave propagation, i.e., of $-\arg \mathbf{G}[x, y]$. The histogram of Fig. 28b indicates a bimodal distribution with a major component pointing upward, i.e., towards the pharynx and a smaller component pointing in opposite direction. This finding is in agreement with the previously determined distribution of the $\mathbf{k}$-vectors (see Fig. 12). However, the present analysis provides also the spatial distribution of the propagation angle $-\arg \mathbf{G}[x, y]$ (see Fig. 28b). Apparently, the upward and downward moving waves are associated with distinct coordination patches. However, the transition between the direction does not coincide with the $\pi$-jump lines. This issue will require a more detailed investigation. In a
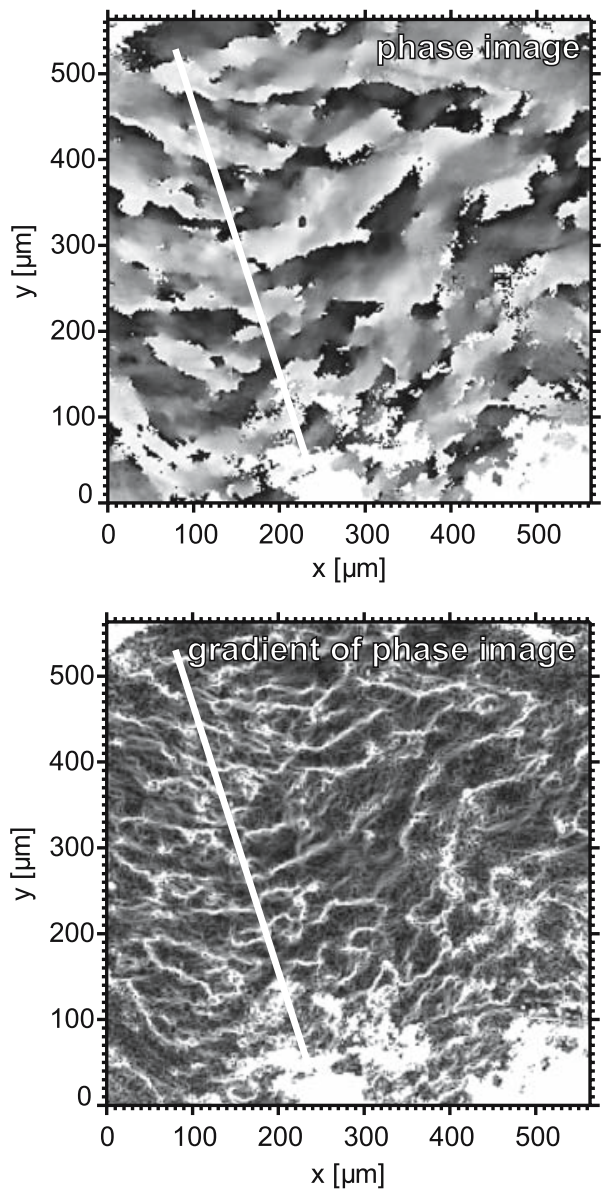

Fig. 25 Gradienting the phase data from a sheep trachea. Lower part shows the modulus of the gradient, i.e., $|\mathbf{G}[x, y, t]|$. The contrast is slightly enhanced by $\gamma=0.7$ to show the defect lines corresponding to $\pi$-jumps together with the background within coordinated patches. Straight line indicates the profiles in Figs. 23 and 26

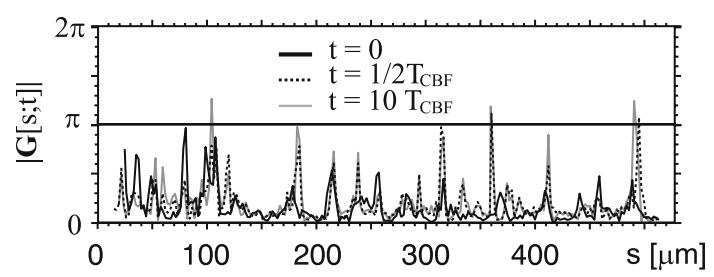

Fig. 26 Line profiles $|\mathbf{G}[s, t]|$ along the white line of the gradient image in Fig. 25; to be compared with Fig. 23. Note that the phase jumps persist over 10 oscillation periods $T_{\mathrm{CBF}}$

majority of patches the waves propagate mainly upward (white areas), that is, in the direction of the pharynx, whereas in others the wave propagation is clearly reversed (black). This apparent lack of long range coordination of the direction of the wave motion is somewhat surprising. How do such poorly coordinated cilia achieve directed mucus transport? 


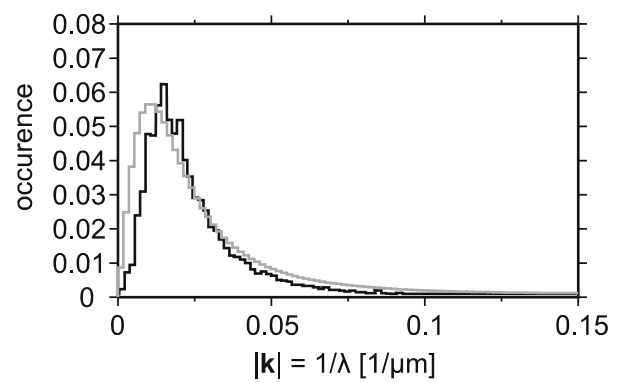

Fig. 27 Histogram of the wave vector distribution $|\mathbf{k}[x, y]|=|\mathbf{G}[x, y]| /$ $2 \pi$ (grey colored line). For comparison, the histogram of the absolute value $|\mathbf{k}|$ from Fig. 12 is also shown (dark line)
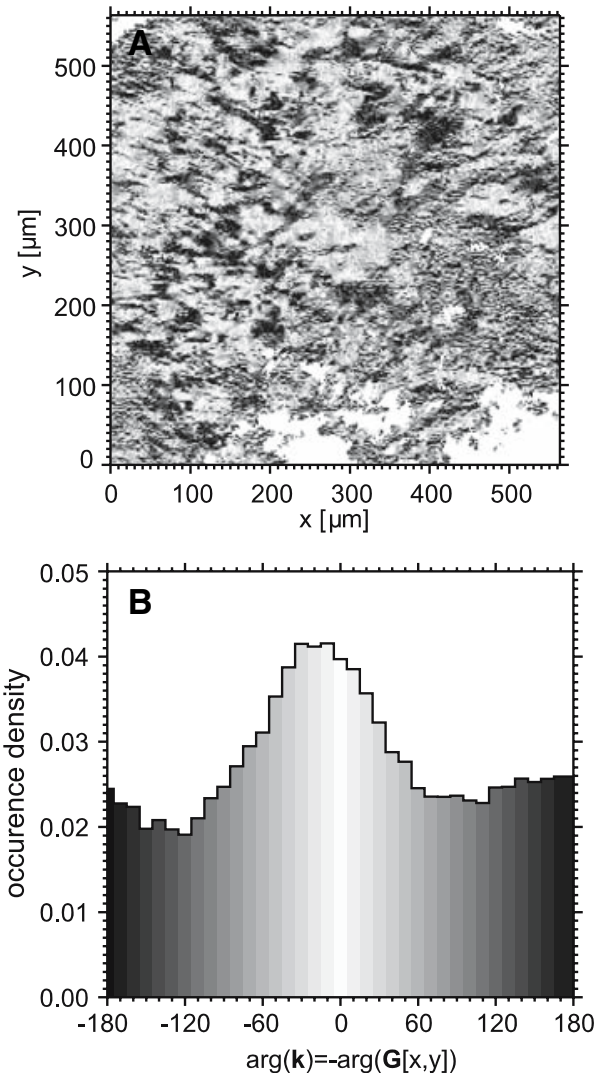

Fig. 28 Spatial distribution of the wave propagation angle $-\arg \mathbf{G}[x, y]$ and the resulting histogram

\section{Mucociliary transport}

The last but not least step of our investigation of the mucociliary phenomena is the analysis of the mucociliary transport. We return to the tracer movies (see Fig. 5d). By tracking the movement of the tracers, we are able to monitor the motion of the mucus. Two-dimensional tracking of sparsely distributed small particles is not difficult. Numerous algorithms can be found in the literature (e.g., Crocker and Grier 1996) and commercial programs

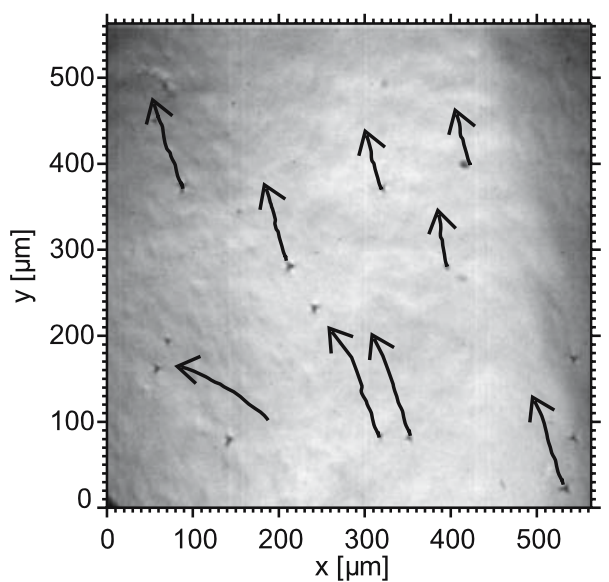

Fig. 29 The tracer paths

are available as well (e.g., DiaTrack, Semasopht, http:// www.semasopht.com). We only recall the basic principles. The tracking consists of two tasks: (1) determination of the tracer positions, and, (2) concatenation of the positions in adjacent frames into unambiguous tracer trajectories. Task (1), the positioning, first requires the determination of the local extrema in a frame of the tracer movie, yielding the tracer positions with the accuracy of one pixel. This accuracy is sufficient for monitoring of the mucus transport and determination of the mean transport velocity. However, the positioning accuracy can be improved if the tracer images are spread over an array of several pixels surrounding the local extremum (in our case an array of $3 \times 3$ pixels). In such a case, one achieves sub-pixel resolution by calculating the centroid or by fitting a model function (a circular step function is sufficient for our purpose) to the luminosity distribution within this pixel array. Task (2), concatenation of the positions into tracer trajectories tends to be more demanding, especially if the observation field is crowded with particles moving in an irregular fashion: Care must be taken to identify unambiguously the particle found in frame $i$ at $\left(x_{i}, y_{i}\right)$ at some new $\left(x_{i+1}, y_{i+1}\right)$ in frame $i+1$. Fortunately in our case the distances between the tracers is large and the overall motion is slow and regular, so that it is sufficient to search for the displaced particle in some small environment surrounding the original position. After a few tracing steps one can estimate the velocity of the tracer and thus predict the expected approximate position in the next frame.

An example of tracing results is given in Fig. 29. From a set of such traces we determine the mean transport velocity $\mathbf{v}$ in the observed area of the tissue. Our findings confirm many previous observations. Typical mucus transport speeds on mammalian tracheas at the temperature of $30^{\circ} \mathrm{C}$ are in the range of $50 \mu \mathrm{m} / \mathrm{s}$, but a large variability is found in different samples and species. In most measurements the 

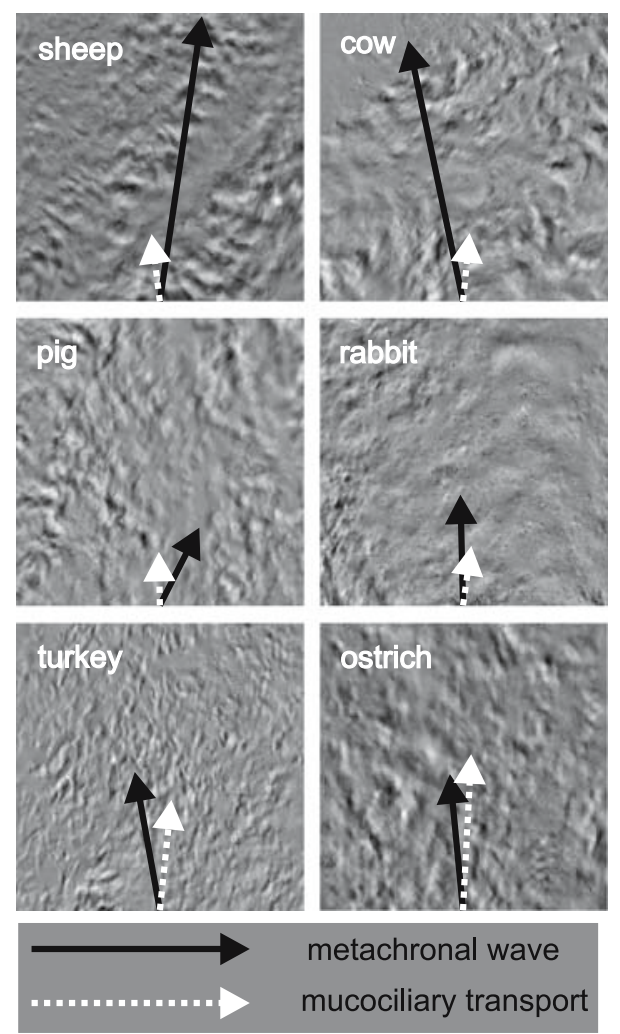

Fig. 30 Illustration of often measured transport directions and mean wave propagation directions. In most measurements, the transport direction nearly coincides with the direction of mean wave propagation

transport direction nearly coincides with the direction of mean wave propagation (as determined from the correlogram), see Fig. 30. These results will be discussed in greater detail in a follow-up publication (Burn et al., submitted). Here we focus on an interesting detail of the tracer trajectories that is currently not understood.

In some of the trajectories the overall straightforward displacement is superposed by circular motion. This feature is found in about $10 \%$ of the trajectories: it is possible that it is especially prevalent in cases with thin mucus layers. An example of a tracer trajectory resolved at the sub-pixel level is shown in Fig. 31, while Fig. 32 shows a sketch of a frequently observed pattern compiled from a number of measured trajectories.

The pattern consists of semi-circular motion. The amplitudes transverse to the transport direction are in the range of 5-6 $\mu \mathrm{m}$ and the amplitudes parallel to the transport direction are in the range of $2-3 \mu \mathrm{m}$. The arc movement may change from clockwise to counterclockwise within a few micrometers. The changeover happens within a typical distance of about $5-10 \mu \mathrm{m}$ and the orientation is maintained over a typical distance of about $50 \mu \mathrm{m}$, corresponding to a typical metachronal wavelength or correlation length. We attempted to correlate the changeover with

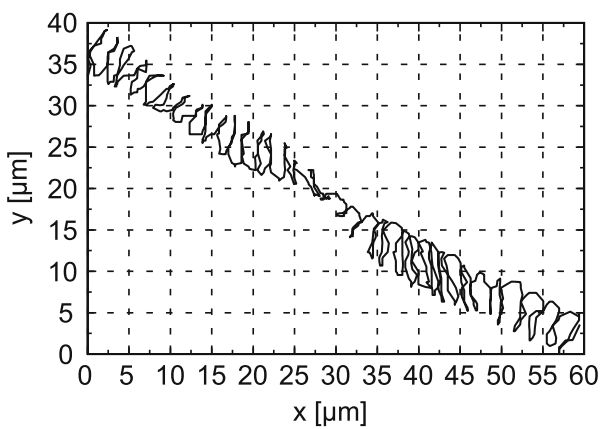

Fig. 31 Details of a particle trace with sub-pixel resolution. The tracer motions have maximal amplitudes of about half a cilium length

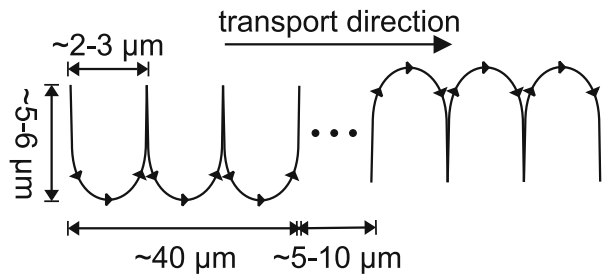

Fig. 32 Schematic sketch of a tracer trajectory frequently observed in the measurement. The sketch is shown with sub-pixel resolution

the locations of the phase jumps and with changes of the wave propagation direction, but so far without success. This interesting effect will require higher spatial resolution of the observation; corresponding measurements are scheduled to be carried out soon.

\section{Conclusions}

We have developed an observation technique and a set of data evaluation tools for a comprehensive characterization of space-time features of mucociliary phenomena on trachea explants. The observation technique is designed for the examination of undisturbed samples under conditions that resemble as closely as possible the native conditions in the respiratory tract. Using an enhanced reflection contrast technique, free of orientation artifacts associated with the traditional Nomarski technique, we detect the modulation of the mucus surface by the underlying ciliary activity simultaneously with the transport of tracer particles embedded in the mucus layer. High-speed digital imaging at $500 \mathrm{fps}$ provides the timing resolution necessary for an unambiguous characterization of the time oscillations. With a spatial resolution of about $2.2 \mu \mathrm{m}$ and an area of observation of $0.5 \times 0.5 \mathrm{~mm}$ we resolve the individual ciliated cells but at the same time obtain an overview over a large area of the tissue containing many cells.

The data evaluation algorithms were designed to extract as completely as possible the large amount of information 
that is available in the three dimensional data sets, i.e., in the recorded movies. We determine simultaneously

- CBF and its distribution on the trachea surface (activity map).

- Mean wavelength of the surface modulation.

- Correlation time of the oscillations and the extent of the spatial correlations.

- Space-time features of phase synchronization.

- Mean velocity of the surface modulation waves, including their mean direction.

- Mean transport velocity and its relation to the wave motion.

These data evaluation tools were applied in a systematic comparative study on mammalian (cow, pig, sheep, rabbit) and bird (ostrich, turkey) tracheae, whose results are reported in (Burn et al., submitted). Here we only summarize the main findings:

The typical CBF values are found in the range between 10 and $15 \mathrm{~Hz}$ (at $30^{\circ} \mathrm{C}$ ) in all investigated species. No significant differences between the species were found, but there is a large variability between the individual samples, the largest measured values being as high as $20 \mathrm{~Hz}$ and the smallest as small as $5 \mathrm{~Hz}$. In contrast, the CBF distribution on the observed surfaces is quite narrow. With a few exceptions the local values deviate by less than $2 \mathrm{~Hz}$ from the mean.

The mean wavelength of the surface modulation of approximately $50 \mu \mathrm{m}$ is found in mammals as well as in birds, corresponding to the size of a couple of ciliated cells. However, we found large variations between the investigated trachea samples of the same species. In some samples we find wavelengths as large as $200 \mu \mathrm{m}$.

While the temporal oscillations remain correlated over a period of time encompassing at least five oscillation periods, the spatial correlation decays within a single wavelength. From the activity maps of the spatial distribution of the CBF we learn that beat frequencies are usually maintained over a much greater range than the spatial correlation length itself. In view of this finding one may speculate that there are two distinct mechanisms controlling ciliary activity: one general clocking mechanism that controls phase differences and a locally acting mechanism controlling the spatial coordination. Both regulation mechanisms may also influence each other, e.g., by coupling via the surrounding mucus or via intercellular signal transduction mechanisms.

Using our algorithm for the space-time analysis of the phase coordination of the ciliary oscillations we found that the spatial de-correlation is mainly due to discrete phase jumps on the order of $1 \pi$. These phase jumps are organized in a web of "defect lines" surrounding patches of more or less coordinated oscillations (see Figs. 24, 25). The struc- ture of the coordination pattern resembles the structures of the patches found in electron micrographs of the ciliated epithelium (Sanderson and Sleigh 1981). The "defect lines" may indicate the boundaries between individual cells, but their spacing is often too large to justify this interpretation.

The speed of the modulation waves is found to be between 100 and $500 \mu \mathrm{m} / \mathrm{s}$. The direction of the wave propagation averaged over the observed area exhibits a large variability, too. The mean angles of propagation with respect to the upward direction (towards the pharynx) are found in a broad range between $-60^{\circ}$ and $60^{\circ}$, but the general tendency is clearly propagation towards the pharynx. Interestingly, the distribution of the propagation directions across the observed surface is bimodal and organized in well discernable patches (see Fig. 28). In the majority of patches the waves tend to propagate upwards but in some of them the propagation is reversed.

Typical mucus transport speeds on mammalian tracheas at a temperature of $30^{\circ} \mathrm{C}$ are in the range of $50 \mu \mathrm{m} / \mathrm{s}$, but a large variability is found in different samples and species. Note that the transport speeds are an order of magnitude smaller than the speeds of the modulation waves (see Fig. 30). Interestingly, the investigated birds (turkey and ostrich) seem to have developed a considerably faster transport systems with a typical speed of $200 \mu \mathrm{m} / \mathrm{s}$, almost as fast as the waves. Not surprisingly, the transport in all species is directed essentially towards the pharynx (i.e., upward), but with quite a large spread of the values in individual samples: the transport angles vary between about $-10^{\circ}$ and $45^{\circ}$ with respect to the upward direction. In all measurements the transport direction is strongly correlated with the direction of mean wave propagation, see Fig. 30.

While some of our results do confirm the observations of previous researchers, we could not confirm the generally accepted picture of antiplectic metachronism, which would mean wave propagation antiparallel to the "effective stroke" (i.e., antiparallel to the transport direction). In our case, the mean propagation direction of the waves is strongly correlated with the transport direction. It is true that we do not observe directly the cilia but rather the modulation of the mucus surface induced by the submucosal metachronal wave. However, considering the small dimensions of the cilia and the small thickness of the surface liquid, only linear coupling between ciliary beating and mucus surface modulation is probable. It is hard to imagine that the surface modulation waves and the submucosal metachronal wave would propagate in opposite direction. The clue to resolve this apparent discrepancy with previous results could be Fig. 28, where we plotted the distribution of the wave propagation direction across the observed tissue area. When examining a small area in the sample, there is a good chance that one selects a 


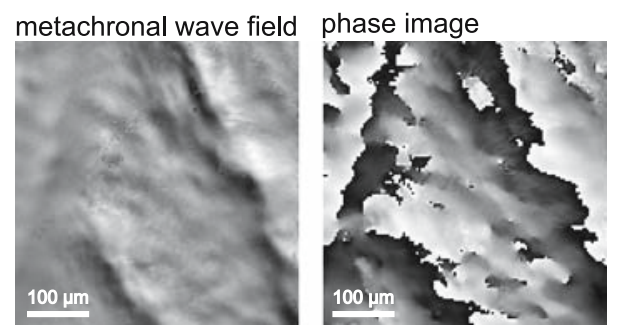

Fig. 33 A singular example of a sheep trachea exhibiting spasmic large scale oscillations but entirely inefficient transport

patch where the waves propagate against the transport direction. Another problem could be the definition of the "effective stroke". In fact, nobody has actually seen the cilia in action, "striking"' the mucus in undisturbed trachea under the mucus blanket.

High resolution observations of the oscillations superposed on the tracer trajectories (see Fig. 31) may shed more light on this problem. Do these oscillations reflect almost directly the motion of the cilia tips? Perhaps, if the tracers penetrate sufficiently deep into the mucus to come in contact with the cilia. However, the magnitude of the maximal oscillation amplitudes is sometimes as large as the full length of a cilium, that is about $7-10 \mu \mathrm{m}$. Since cilia in the respiratory epithelium are densely packed, it is hard to believe that they are capable of such a large stroke. It is conceivable that the tracer oscillations reflect a resonant excitation of an oscillating mode in the visco-elastic mucus layer. This would indicate the importance of the rheological properties of the mucus in the functioning of the mucociliary escalator.

In any case, despite the long history of research, the mucociliary transport remains puzzling. A particular puzzle is, for example, the large variability of the phenomena. A singular extreme example is the movie S7 from a sheep trachea provided in the supplementary material (an image out of the movie is shown in Fig. 33): note the overcoordinated metachronism, exhibiting spasmic large scale oscillations but entirely inefficient transport. Is a certain degree of "metachronal wave disorder" necessary to achieve efficient transport? What makes those cilia behave in such a strange way, and what would we learn if we knew the reason for their strange behavior?

Acknowledgments The work was partly supported by the Swiss National Science Foundation. We thank Patrick Stoller for kindly proofreading the manuscript.

\section{References}

Ben-Shimol Y, Dinstein I, Meisels A, Priel Z (1991) Ciliary motion features from digitized video photography. J Comput Assist Microsc 3(3):103-116
Bogdanovic N, Krattiger B, Ricka J, Frenz M (2005) Apparatus for endoscopical, laser-based determination of ciliary beat frequency. In SPIE: novel optical instrumentation for biomedical applications II 5864:27-38

Bonnaire Y, Dubreuil A, Bouley G, Boudene C (1980) An improved device for frequency recording of tracheal ciliary beat. Microsc Acta 83(3):221-227

Burn A, Ryser M, Wessel T, Ricka J, Frenz M Functional imaging of muco-ciliary phenomena-an inter-species comparative study. (Submitted)

Chandra T, Yeates DB, Miller IF, Wong LB (1994) Stationary and nonstationary correlation-frequency analysis of heterodyne mode laser-light scattering-magnitude and periodicity of canine tracheal ciliary beat frequency in-vivo. Biophys J 66(3):878-890

Chilvers MA, O'Callaghan C (2000) Analysis of ciliary beat pattern and beat frequency using digital high speed imaging: comparison with the photomultiplier and photodiode methods. Thorax 55(4):314-317

Crocker JC, Grier DG (1996) Methods of digital video microscopy for colloidal studies. J Colloid Interface Sci 179(1):298-310

Curtis LN, Lea SM, Muir RB, Carson JL (1991) Measurement of ciliary beat frequency using a computer-assisted photometric video technique: Correlation of fast fourier transform (fft) analysis to ciliary beating. J Comput Assist Microsc 3(2):65-76

Dimova S, Maes F, Brewster ME, Jorissen M, Noppe M, Augustijns P (2005) High-speed digital imaging method for ciliary beat frequency measurement. J Pharm Pharmacol 57(4):521-526

Dresdner RD, Wong LB (1985) Measurement of ciliary beat frequency using high-speed video microscopy. ISA Trans 24(1):33-38

Gheber L, Priel Z (1989) Synchronization between beating cilia. Biophys J 55(1):183-191

Gheber L, Priel Z (1994) Metachronal activity of cultured mucociliary epithelium under normal and stimulated conditions. Cell Motil Cytoskeleton 28(4):333-345

Gray J (1930) The mechanism of ciliary movement-via photographic and stroboscopic analysis of ciliary movement. Proc R Soc Lond B 107(751):313-332

Hennessy SJ, Wong LB, Yeates DB, Miller IF (1986) Automated measurement of ciliary beat frequency. J Appl Physiol 60(6):2109-13

Jacobsen E, Lyons R (2003) The sliding dft. Signal Processing Mag IEEE 20(2):74-80

James WC, John WT (1965) An algorithm for the machine calculation of complex fourier series. Math Comput 19:297-301

Joki S, Saano V (1994) Ciliary beat frequency at six levels of the respiratory tract in cow, dog, guinea-pig, pig, rabbit and rat. Clin Exp Pharmacol Physiol 21(5):427-434

Korngreen A, Priel Z (1994) Simultaneous measurement of ciliary beating and intracellular calcium. Biophys J 67(1):377-80

Lee JS (1986) Speckle suppression and analysis for synthetic aperture radar images. Opt Eng 25(5):636-634

Lee WI, Verdugo P (1976) Laser light-scattering spectroscopy-new application in study of ciliary activity. Biophys J 16(9):11151119

Lee CH, Lee SS, Mo JH, Kim IS, Quan SH, Wang SY, Yi WJ, Rhee CS, Min YG (2005) Comparison of ciliary wave disorders measured by image analysis and electron microscopy. Acta Otolaryngol 125(5):571-576

Lucas AM (1932) Coordination of ciliary movement in methods of study and the relation of ciliary coordination to ciliary inhibition. J Morphol 53(2):243-263

Ovadyahu D, Priel Z (1989) Characterization of metachronal wave in beating cilia-distribution of phases in space. Biorheology 26(4):677-685 
Press WH, Flannery BP, Teukolsky SA, Vettering WT (1992) Numerical recipes in $\mathrm{C}$ : the art of scientific computing. Cambridge University Press, Cambridge

Priel Z (1987) Direct measurement of the velocity of the metachronal wave in beating cilia. Biorheology 24(6):599-603

Purkinje JE, Valentin G (1834) Entdeckung continuirlicher durch Wimperhaare erzeugter Flimmerbewegungen, als eines allgemeinen Phänomens in den Klassen der Amphibien, Vögel und Säugethiere. Arch Anat Physiol U Wiss Med (Müllers Archiv) 36:391-398

Rautiainen M, Matsune S, Shima S, Sakamoto K, Hanamure Y, Ohyama M (1992) Ciliary beat of cultured human respiratory cells studied with differential interference microscope and highspeed video system. Acta Otolaryngol 112(5):845-851

Roberts LG (1965) Machine perception of three-dimensional solids. Optical and electro-optical information processing. MIT Press, Cambridge, pp 159-197

Romet S, Schoevaert D, Marano F (1991) Dynamic image-analysis applied to the study of ciliary beat on cultured ciliated epithelialcells from rabbit trachea. Biol Cell 71(1-2):183-190

Salathe M, Bookman RJ (1995) Coupling of [ca2+]i and ciliary beating in cultured tracheal epithelial cells. J Cell Sci 108(Pt 2):431-40

Sanderson MJ (2000) High-speed digital microscopy. Methods 21(4):325-334

Sanderson MJ, Sleigh MA (1981) Ciliary activity of cultured rabbit tracheal epithelium-beat pattern and metachrony. J Cell Sci 47:331-347

Sanderson MJ, Charles AC, Dirksen ER (1990) Mechanical stimulation and intercellular communication increases intracellular ca2+ in epithelial cells. Cell Regul, 1(8):585-96, 1044-2030 (Print) Journal Article

Satir P, Sleigh MA (1990) The physiology of cilia and mucociliary interactions. Annu Rev Physiol 52:137-155
Schipor I, Palmer JN, Cohen AS, Cohen NA (2006) Quantification of ciliary beat frequency in sinonasal epithelial cells using differential interference contrast microscopy and high-speed digital video imaging. Am J Rhinol 20(1):124-127

Sisson JH, Stoner JA, Ammons BA, Wyatt TA (2003) All-digital image capture and whole-field analysis of ciliary beat frequency. J Microsc 211:103-111

Sleigh MA, Blake JR, Liron N (1988) The propulsion of mucus by cilia. Am Rev Respir Dis 137(3):726-41

Svartengren K, Wiman LG, Thyberg P, Rigler R (1989) Laser light scattering spectroscopy: a new method to measure tracheobronchial mucociliary activity. Thorax 44(7):539-547

Teichtahl H, Wright PL, Kirsner, RLG (1986) Measurement of invitro ciliary beat frequency-a television-video modification of the transmitted light technique. Med Biol Eng Comput 24(2):193196

Wanner A, Salathe M, O'Riordan TG (1996) Mucociliary clearance in the airways. Am J Respir Crit Care Med 154:1868-1902

Wessel Th, Frenz M, Ricka J (1999) Measurement of frequency and metachronal wavelength of ciliary beating by dynamic light scattering and differential interference contrast microscopy. In Biomedical optics: new concepts in therapeutic laser applications, novel biomedical optical spectroscopy, imaging, and diagnostics, advances in optical imaging, photon migration, and tissue optics, pp 178-180

Wong LB, Miller IF, Yeates DB (1993) Nature of the mammalian ciliary metachronal wave. J Appl Physiol 75(1):458-467

Yi WJ, Park KS, Lee CH, Rhee CS, Nam SW (2002) Directional disorder of ciliary metachronal waves using two-dimensional correlation map. IEEE Trans Biomed Eng 49(3):269-273

Yi WJ, Park KS, Lee CH, Rhee CS (2003) Correlation between ciliary beat frequency and metachronal wave disorder using image analysis method. Med Biol Eng Comput 41(4):481-485 